\title{
First supplement to the catalogue of invertebrate and vertebrate palaeontological type specimens of the Hungarian Natural History Museum: 2008-2018
}

\author{
Alfréd Dulai ${ }^{1}$, Mihály GaspariK ${ }^{1}$, Zoltán Szentesi ${ }^{1} \&$ József PÁLFY $^{2,3}$ \\ ${ }^{1}$ Department of Palaeontology and Geology, Hungarian Natural History Museum, \\ H-1083 Budapest, Ludovika tér 2, Hungary.E-mails: dulai.alfred@nhmus.hu, \\ gasparik.mihaly@nhmus.hu,szentesi.zoltan@nhmus.hu; \\ ${ }^{2}$ MTA-MTM-ELTE Research Group for Palaeontology, \\ H-1083 Budapest, Ludovika tér 2, Hungary. \\ E-mail:palfy.jozsef@nhmus.hu; \\ ${ }^{3}$ Department of Geology, Eötvös Loránd University, \\ H-1117 Budapest, Pázmány Péter sétány 1/C, Hungary. \\ E-mail:palfy@elte.hu
}

\begin{abstract}
Summary - A comprehensive palaeontological type catalogue of the Hungarian Natural History Museum (Budapest) was published in 2008. In the following ten years several new type specimens were deposited in the collection, both invertebrates and vertebrates, from microscopic radiolarians to large dinosaur bone remains. Triassic radiolarians and ammonoids, Jurassic brachiopods, Cenozoic molluscs, and Cretaceous vertebrates represent the most dynamically growing parts of the type collection. This supplement to the original catalogue contains the data of 175 species and subspecies, of which 148 are new taxa for the type catalogue. Among them 125 are represented by holotypes, and an additional 199 inventory lots of their paratypes; 23 new taxa are represented only by paratypes, in an additional 74 inventory lots. The vast majority of these taxa were recently described (between 2008 and 2018), although a few of them are previously established taxa with type material that was newly recognized to exist in our collection. This supplement also contains the data of four recently designated neotypes, as well as 16 rediscovered old type specimens which were reported as missing in the 2008 catalogue. We also include the known revisions and illustration of 14 previously established species and subspecies (in three cases with lectotype designation). At the end of 2018, the invertebrate and vertebrate palaeontological collection of the Hungarian Natural History Museum holds more than 2500 inventory lots of different type specimens, including name-bearing types of 1056 nominal species and subspecies.
\end{abstract}

Key words - holotype, invertebrates, lectotype, neotype, new taxa, palaeontology, paratype, vertebrates 


\section{TABLE OF CONTENTS}

Introduction

$\begin{array}{lr}\text { Catalogue of invertebrate type specimens } & 10\end{array}$

$\begin{array}{ll}\text { 1. Triassic types } & 10\end{array}$

1.1. Triassic Radiolaria $\quad 10$

1.2. Triassic Gastropoda $\quad 18$

1.3. Triassic Cephalopoda $\quad 18$

2. Jurassic types 21

2.1. Jurassic Bivalvia 21

2.2. Jurassic Gastropoda $\quad 22$

2.3. Jurassic Cephalopoda 24

2.4. Jurassic Brachiopoda 25

3. Cretaceous types 26

3.1. Cretaceous Cephalopoda 26

3.2. Cretaceous Brachiopoda 26

3.3. Cretaceous Crinoidea 26

3.4. Cretaceous Echinoidea 27

4. Eocene types 27

4.1. Eocene Foraminiferida 27

4.2. Eocene Polyplacophora 28

4.3. Eocene Brachiopoda 28

5. Oligocene types 29

5.1. Oligocene Gastropoda $\quad 29$

5.2. Oligocene Decapoda 29

6. Miocene types $\quad 32$

6.1. Miocene Bivalvia $\quad 32$

6.2. Miocene Gastropoda $\quad 32$

6.3. Miocene Brachiopoda 34

6.4. Miocene Decapoda 34

6.5. Miocene Echinoidea 34

Catalogue of vertebrate type specimens $\quad 35$

7. Amphibia 35

8. Reptilia 36

9. Aves 46

10. Mammalia 46

Index of species and subspecies names $\quad 49$

References $\quad 52$ 


\section{INTRODUCTION}

After moving of the palaeontological collections to a new location in 20042006, and the development of a digital collection database, the first catalogue of palaeontological type specimens of the Hungarian Natural History Museum (HNHM) was published by PÁlfy et al. (2008). Some additional data on the types and the collections were summarized by PÁLFY (2009). The history and development of the type catalogue was described in detail in PÁLFY et al. (2008). The catalogue provided a comprehensive listing of invertebrate and vertebrate type specimens published between 1841 and February 2008. The palaeobotanical type specimens are historically part of the collection of the Department of Botany at the HNHM (HABLY \& SzAKÁly 1989; HABLY et al. 2001).

The catalogue listed type specimens of 1181 fossil species-group taxa (986 invertebrates and 195 vertebrates). Discounting the specimens that are missing, lost or have been transferred to other institutions, the catalogue included primary types of 919 taxa available in the collection of the HNHM. At that time 2017 existing inventory lots contained type specimens.

The present work supplements PÁLFY et al's (2008) catalogue, including the type specimens published between February 2008 and December 2018, as well as those specimens which were regarded as missing types in the original catalogue, but later were rediscovered in the collection. We also include those previously published types which were revised or re-illustrated between 2008 and 2018. Several type specimens were recently discovered in some historical collections or older materials, or were donated to the HNHM (e.g., PAPP 1907; Kutassy 1933; SOMOS \& KóKAY 1960; STEININGER et al. 1973).

Following the structure of the original catalogue, this supplement also includes primary, name-bearing types (holotypes, syntypes, lectotypes, neotypes) and secondary types (paratypes, paralectotypes). It is subdivided into separate parts for invertebrate and vertebrate types. The basic information of taxa and their type specimens is listed in slightly different formats for invertebrates and vertebrates. An individual catalogue entry typically corresponds to an inventory lot that contains the type specimen(s) of a taxon, identified by an inventory number. If a taxon is represented by more than one type specimen, separate entries are listed where differences exist in either the inventory number, locality, or illustration of specimen. Types under more than one inventory number are merged into a single entry if all data fields other than the inventory number are identical. 
The format for invertebrate entries is as follows:

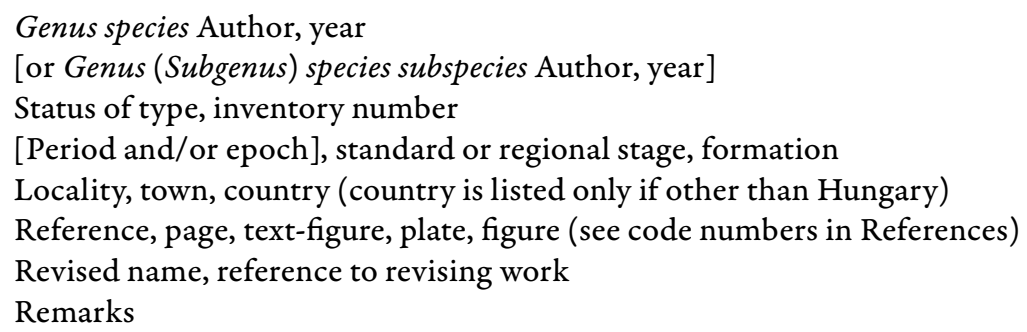

The format for pre-Pliocene vertebrate entries is as follows:

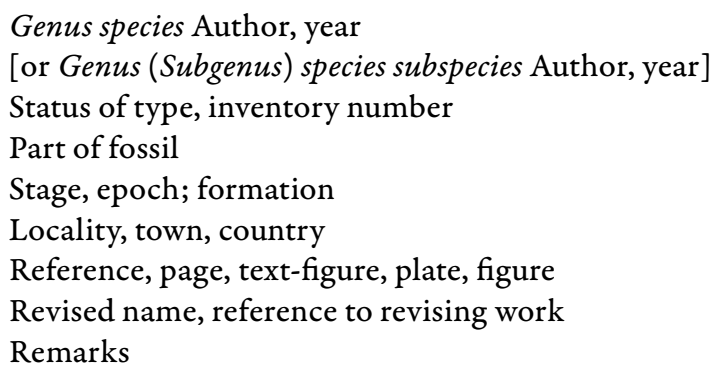

The format for Pliocene and Quaternary vertebrate entries is as follows:

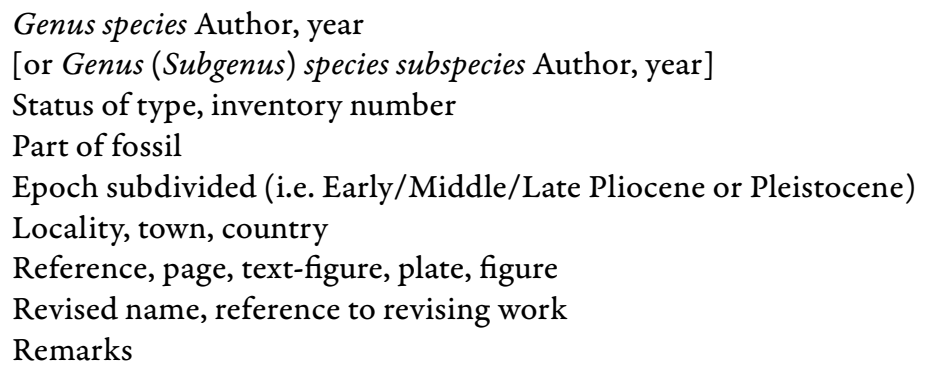

The invertebrate catalogue entries are arranged primarily by geologic age, secondarily by major taxonomic groups, and then the original proposed bi- or trinomens are listed alphabetically within each subdivision.

Both the ages and the taxonomic groups that define the subdivisions were selected for practical reasons rather than strictly using certain levels in their hierarchical structure. The following age subdivisions, ranging in rank from period to epoch, are used: Triassic, Jurassic, Cretaceous, Eocene, Oligocene, and Miocene. Secondary, taxonomic subdivisions use the following, well-known groups, arbitrarily taken from higher taxa at the phylum, class, or order level: Radiolaria, Foraminiferida, Polyplacophora, Bivalvia, Gastropoda, Cephalopoda, Brachiopoda, Decapoda, Crinoidea, Echinoidea.

The catalogue of vertebrate types is arranged by higher taxa, containing subdivisions for Amphibia, Reptilia, Aves, and Mammalia. 
Taxon names for the types are given as bi- or trinomens as they were introduced by the author in the original description. The original spelling is retained, even though current rules of ICZN (see Article 32) require avoiding the diacritical marks of non-standard Latin characters (commonly occurring in taxon names derived from Hungarian person or place names). However, preserving the original spelling in the catalogue may help tracing a taxon in the literature. Necessary corrections and emendations of originally incorrect spelling of species names are reflected in the index of species and subspecies names.

If types of a taxon require more than one entry, the holotype or lectotype is listed first. For types of equal status (e.g. syntypes, paratypes, paralectotypes), the entries are arranged in ascending order of their inventory number. In merged entries with several specimens of identical attributes, the inventory numbers are given in a list separated by commas.

If possible, an age assignment for invertebrate specimens is given at the stage level. Standard stages are used (Gradstein et al. 2012) except for the Oligocene and Miocene, where the regional stages of the Central Paratethys time scale are applied (PILlER et al. 2007). Although some stages are known to straddle standard epoch boundaries, for sake of a simplified hierarchy of age units, these boundary problems are sidestepped. Thus the Egerian is included in the Oligocene, and the Pannonian is taken to form part of the Miocene. Original age assignments are revised if necessary, where up-to-date stratigraphic information is available from the type locality.

The ages of Pliocene and Pleistocene vertebrates are given using a threefold (i.e. Early-Middle-Late) subdivision of the epochs, derived by simplifying the biochronological framework of KRETzor (1969) and JÁnOssy (1986), with the necessary modifications to account for different concepts in placing the Pliocene/Pleistocene boundary. Age assignment of pre-Pliocene vertebrates follows the concepts applied to invertebrates.

The lithostratigraphic unit from which a type specimen was collected is given on the basis of CsÁszÁR (1997) for Hungary; in other countries the relevant references were consulted for information on lithostratigraphy. Pliocene and younger vertebrates are not assigned to formations as they are most commonly found in fissure fills and cave deposits, not forming conventional lithostratigraphic units.

In the locality field, geographic names are given in their usual Hungarian form, for ease of finding them on local maps. The English translation of some common vernacular terms in geographic names (often hyphenated to form the latter part of a proper name) is listed as follows (Hungarian words in italics): árok - gully; domb - hill; hegy - mountain; kút - well; mezö - meadow; puszta - farm; völgy - valley. Geological terms for localities, such as outcrop (“feltárás”), quarry 
(“kőfejtô"), brickyard ("téglagyár"), coal mine ("szénbánya") are given in English. Name of town refers to the municipality to which the locality belongs, in some cases this is not the nearest settlement.

As the majority of the types were collected at localities within the presentday borders of Hungary ( 385 inventory lots), no country name is listed for them. Some specimens are from sites in historical Hungary that are now in the territory of Slovakia (7) or Romania (1). Their town is listed using the current official name, with the Hungarian name given in parentheses. Approximately one-quarter of the types are from other countries, including Turkey (97), Austria (16), Russia (8), France (8), and Poland (3).

Reference is given to the original description, using a five or six character code, found in the reference list at the end of each item. The reference code is composed of the first three letters of the first author's name (or an otherwise meaningful three-letter abbreviation of the name), the last two digits of the year of publication, and, if needed, an extra letter to distinguish works from the same year. If the original description appeared in Hungarian as well as in a foreign language, both page numbers are given, the foreign one first and the Hungarian one in parentheses.

Illustrations (text-figures and/or figures on plates) accompanying the original description are quoted at the appropriate entry, if the specimen is identified as the figured one.

If we became aware of a published revision, either nomenclatural, or changing the species-level identification or the generic assignment of the taxon concerned, we include the revised name and provide the reference code of the revising work.

Any additional information may appear as remarks in the last line of an item. Remarks may include reference to a different inventory number in the original publication, notes on the type designation, or the date of rediscovery in the collection.

To facilitate finding information on taxa, the catalogue is followed by an index of all invertebrate and vertebrate species and subspecies names. Here an original name (using emended spelling if needed) is followed by the originally published bi- or trinomen, the author's name, and the page number on which the first catalogue entry of this taxon appears.

Although significant care was taken to compile a comprehensive catalogue that contains accurate information, errors and omissions are almost inevitable in an endeavour of this scale. Therefore users of this catalogue are encouraged to report any suggested correction and/or addition to the curators of the Department of Palaeontology and Geology. 
Acknowledgements - First and foremost, special thanks to Zsuzsanna Molnár for her enormous effort to sift through the relevant literature, as well as for her dedicated work in data entry and editing. Our active and retired colleagues in the Department of Palaeontology and Geology (István Főzy, Ottilia Szives, Attila Vörös, János Szabó) provided useful help in the collection under their care. Additional useful information on specimens and references were provided by Péter Ozsvárt, Attila Ősi, Piroska Pazonyi, Attila Virág, Matúš Hyžný, and Günther Schweigert. Access to the Mesozoic vertebrate collection was facilitated by Réka Kalmár. Eszter Hankó helped with data entry to the inventory. 


\section{Catalogue of invertebrate type specimens}

\section{Triassic types}

\subsection{Triassic Radiolaria}

Annulohaeckeliella curvispina Ozsvárt, Dumitrica \& Hungerbühler in OzsváRT et al., 2017 holotype, PAL 2016.22.1.

Carnian, Killik Formation

Tavusçayırı Tepe, Sorgun, Turkey

Ozs17a, p. 154, pl. 3, fig. 9

Archaeosemantis multispinosa Ozsvárt, Dumitrica \& Moix, 2017

holotype, PAL 2017.9.2.

Carnian, Killik Formation

Tavusçayırı Tepe, Sorgun, Turkey

Ozs17b, p. 65, fig. 7.11

No inventory number given in original publication

Archaeosemantis multispinosa Ozsvárt, Dumitrica \& Moix, 2017

paratype, PAL 2017.9.1.

Carnian, Killik Formation

Tavusçayırı Tepe, Sorgun, Turkey

Ozs17b, p. 65, fig. 7.10

No inventory number given in original publication

Archaeosemantis multispinosa Ozsvárt, Dumitrica \& Moix, 2017

paratype, PAL 2017.9.3.

Carnian, Killik Formation

Tavusçayırı Tepe, Sorgun, Turkey

Ozs17b, p. 65, fig. 7.12

No inventory number given in original publication

Baumgartneria szarvaskoensis Ozsvárt \&

Kovács, 2012

holotype, PAL 2018.24.1.

Ladinian, Extra-Bükkian olistoliths

Malom-hegy, Szarvaskő

Ozs12, p. 281, pl. 2, figs 19-20

No inventory number given in original publication
Baumgartneria szarvaskoensis Ozsvárt \&

Kovács, 2012

paratype, PAL 2018.25.1.

Ladinian, Extra-Bükkian olistoliths

Malom-hegy, Szarvaskő

Ozs12, p. 281, pl. 2, fig. 21

No inventory number given in original publi-

cation

Baumgartneria szarvaskoensis Ozsvárt \&

Kovács, 2012

paratype, PAL 2018.25.2.

Ladinian, Extra-Bükkian olistoliths

Malom-hegy, Szarvaskő

Ozs12, p. 281, pl. 2, fig. 22

No inventory number given in original publication

Capnuchosphaera barnabasi Kozur, Moix \&

Ozsvárt, 2009

holotype, M 2009.1.1.

Carnian, Killik Formation

Tavusçayırı Tepe, Sorgun, Turkey

Koz09, p. 32, pl. 2, fig. 1

Capnuchosphaera borbalae Kozur, Moix \&

Ozsvárt, 2009

holotype, M 2009.3.1.

Carnian, Killik Formation

Tavusçayırı Tepe, Sorgun, Turkey

Koz09, p. 32, pl. 1, fig. 2

Capnuchosphaera bragini Kozur, Moix \&

Ozsvárt, 2009

holotype, M 2009.4.1.

Carnian, Killik Formation

Tavusçayırı Tepe, Sorgun, Turkey

Koz09, p. 32, pl. 1, fig. 7

Capnuchosphaera ciliciensis Kozur, Moix \&

Ozsvárt, 2009

holotype, M 2009.6.1.

Carnian, Killik Formation

Tavusçayırı Tepe, Sorgun, Turkey

Koz09, p. 33, pl. 2, fig. 4 
Capnuchosphaera crassa yforma Kozur, Moix \& Ozsvárt, 2009

holotype, M 2009.9.1.

Carnian, Killik Formation

Tavusçayırı Tepe, Sorgun, Turkey

Koz09, p. 33, pl. 2, fig. 7

Capnuchosphaera cylindrica cylindrica Kozur, Moix \& Ozsvárt, 2009

holotype, M 2009.10.1.

Carnian, Killik Formation

Tavusçayırı Tepe, Sorgun, Turkey

Koz09, p. 33, pl. 1, fig. 10

Capnuchosphaera cylindrica retusaspinosa

Kozur, Moix \& Ozsvárt, 2009

holotype, M 2009.11.1.

Carnian, Killik Formation

Tavusçayırı Tepe, Sorgun, Turkey

Koz09, p. 34, pl. 1, fig. 11

Capnuchosphaera goestlingensis Kozur, Mostler \& Ozsvárt in Kozur et al., 2009

paratype, M 2009.12.1.

Carnian, Göstling Formation

Göstling, Austria

Koz09, p. 34, pl. 2, fig. 11

Capnuchosphaera gracilispinosa gracilispinosa

Kozur, Moix \& Ozsvárt, 2009

holotype, M 2009.13.1.

Carnian, Killik Formation

Tavusçayırı Tepe, Sorgun, Turkey

Koz09, p. 34, pl. 1, fig. 4

Capnuchosphaera gracilispinosa turkensis Kozur, Moix \& Ozsvárt, 2009

holotype, M 2009.14.1.

Carnian, Killik Formation

Tavusçayırı Tepe, Sorgun, Turkey

Koz09, p. 34, pl. 1, fig. 5

Capnuchosphaera mersinensis Kozur, Moix \&

Ozsvárt, 2009

holotype, M 2009.16.1.

Carnian, Killik Formation

Tavusçayırı Tepe, Sorgun, Turkey

Koz09, p. 35, pl. 1, fig. 3
Capnuchosphaera mostleri Kozur, Moix \&

Ozsvárt, 2009

holotype, M 2009.17.1.

Carnian, Killik Formation

Tavusçayırı Tepe, Sorgun, Turkey

Koz09, p. 35, pl. 1, fig. 9

Capnuchosphaera multispinosa Kozur, Moix \&

Ozsvárt, 2009

holotype, M 2009.18.1.

Carnian, Killik Formation

Tavusçayırı Tepe, Sorgun, Turkey

Koz09, p. 35, pl. 3, fig. 2

Capnuchosphaera oesii Kozur, Moix \& Ozsvárt, 2009

holotype, M 2009.20.1.

Carnian, Killik Formation

Tavusçayırı Tepe, Sorgun, Turkey

Koz09, p. 36, pl. 1, fig. 12

Capnuchosphaera ottomanensis ottomanensis

Kozur, Moix \& Ozsvárt, 2009

holotype, M 2009.21.1.

Carnian, Killik Formation

Tavusçayırı Tepe, Sorgun, Turkey

Koz09, p. 36, pl. 2, fig. 8

Capnuchosphaera ottomanensis carterae Kozur, Moix \& Ozsvárt, 2009

holotype, M 2009.23.1.

Carnian, Killik Formation

Tavusçayırı Tepe, Sorgun, Turkey

Koz09, p. 36, pl. 2, fig. 10

Capnuchosphaera tortuospinosa Kozur, Moix \& Ozsvárt, 2009

holotype, M 2009.24.1.

Carnian, Killik Formation

Tavusçayırı Tepe, Sorgun, Turkey

Koz09, p. 36, pl. 1, fig. 1

Capnuchosphaera tumorspinosa Kozur, Moix \& Ozsvárt, 2009

holotype, M 2009.25.1.

Carnian, Killik Formation

Tavusçayırı Tepe, Sorgun, Turkey

Koz09, p. 37, pl. 3, fig. 1 
Capnuchosphaera tuvalica Kozur, Moix \&

Ozsvárt, 2009

holotype, M 2009.26.1.

Carnian, Killik Formation

Tavusçayırı Tepe, Sorgun, Turkey

Koz09, p. 37, pl. 2, fig. 12

Caponabolella brevispinosa Ozsvárt, Dumitrica

\& Hungerbühler in OzsváRT et al., 2017

holotype, PAL 2016.27.1.

Carnian, Killik Formation

Tavusçayırı Tepe, Sorgun, Turkey

Ozs17a, p. 158, pl. 5, fig. 6

Caponabolella brevispinosa Ozsvárt, Dumitrica

\& Hungerbühler in OzsvÁRT et al., 2017

paratype, PAL 2016.27.2.

Carnian, Killik Formation

Tavusçayırı Tepe, Sorgun, Turkey

Ozs17a, p. 158, pl. 5, fig. 7

No inventory number given in original publication

Caponabolella longispinosa Ozsvárt, Dumitrica \& Hungerbühler in OzsvÁRT et al., 2017

holotype, PAL 2016.26.1.

Carnian, Killik Formation

Tavusçayırı Tepe, Sorgun, Turkey

Ozs17a, p. 156, pl. 5, figs 1-2

Caponabolella longispinosa Ozsvárt, Dumitrica \& Hungerbühler in OzsvárT et al., 2017

paratype, PAL 2016.26.2.

Carnian, Killik Formation

Tavusçayırı Tepe, Sorgun, Turkey

Ozs17a, p. 156, pl. 5, fig. 3

No inventory number given in original publication

Circopoulpus cornubovis Ozsvárt, Dumitrica \& Hungerbühler in OzsvÁRT et al., 2017

holotype, PAL 2016.21.1.

Carnian, Killik Formation

Tavusçayırı Tepe, Sorgun, Turkey

Ozs17a, p. 149, pl. 2, fig. 12
Circopoulpus cornubovis Ozsvárt, Dumitrica \& Hungerbühler in Ozsvárt et al., 2017

paratype, PAL 2016.21.3.

Carnian, Killik Formation

Tavusçayırı Tepe, Sorgun, Turkey

Ozs17a, p. 149, pl. 2, fig. 9

No inventory number given in original publication

Circopoulpus cornubovis Ozsvárt, Dumitrica \& Hungerbühler in OzsvárT et al., 2017

paratype, PAL 2016.21.4.

Carnian, Killik Formation

Tavusçayırı Tepe, Sorgun, Turkey

Ozs17a, p. 149, pl. 2, fig. 10

No inventory number given in original publication

Circopoulpus cornubovis Ozsvárt, Dumitrica \& Hungerbühler in OzsváRT et al., 2017

paratype, PAL 2016.21.5.

Carnian, Killik Formation

Tavusçayırı Tepe, Sorgun, Turkey

Ozs17a, p. 149, pl. 2, fig. 11

No inventory number given in original publication

Circopoulpus dulaii Ozsvárt, Dumitrica \&

Hungerbühler in OzsvárT et al., 2017

paratype, PAL 2016.20.1.

Carnian, Killik Formation

Tavusçayırı Tepe, Sorgun, Turkey

Ozs17a, p. 149, pl. 2, fig. 7

No inventory number given in original publication

Goricanella hexaspinosa Kozur, Mostler \&

Ozsvárt in Kozur et al., 2009

holotype, M 2009.47.1.

Carnian, Göstling Formation

Göstling, Austria

Koz09, p. 43, pl. 4, fig. 14

Goricanella hexaspinosa Kozur, Mostler \&

Ozsvárt in Kozur et al., 2009

paratype, M 2009.47.2.

Carnian, Göstling Formation

Göstling, Austria

Koz09, p. 43, pl. 4, fig. 15 
Goricanella hexaspinosa Kozur, Mostler \&

Ozsvárt in Kozur et al., 2009

paratype, M 2009.47.3.

Carnian, Göstling Formation

Göstling, Austria

Koz09, p. 43, pl. 4, fig. 16

Hindeosphaera burrii Ozsvárt, Moix \& Kozur, 2015

holotype, PAL 2014.136.1.

Carnian, Killik Formation

Tavusçayırı Tepe, Sorgun, Turkey

Ozs15, p. 346, fig. 5.23

Hindeosphaera burrii Ozsvárt, Moix \& Kozur, 2015

paratype, PAL 2014.136.2.

Carnian, Killik Formation

Tavusçayırı Tepe, Sorgun, Turkey

Ozs15, p. 346, fig. 5.22

Hindeosphaera burrii Ozsvárt, Moix \& Kozur, 2015

paratype, PAL 2014.136.3.

Carnian, Killik Formation

Tavusçayırı Tepe, Sorgun, Turkey

Ozs15, p. 346, fig. 5.21

Hindeosphaera djani Ozsvárt, Moix \& Kozur, 2015

holotype, PAL 2014.135.1.

Carnian, Killik Formation

Tavusçayırı Tepe, Sorgun, Turkey

Ozs15, p. 346, fig. 5.20

Hindeosphaera naomiae Ozsvárt, Moix \&

Kozur, 2015

holotype, PAL 2014.134.1.

Carnian, Killik Formation

Tavusçayırı Tepe, Sorgun, Turkey

Ozs15, p. 348, fig. 5.19

Minicrampus longispinosus Ozsvárt, Dumitrica

\& Moix, 2017

holotype, PAL 2017.5.1.

Carnian, Killik Formation

Tavusçayırı Tepe, Sorgun, Turkey

Ozs17b, p. 62, fig. 7.1

No inventory number given in original publication
Minicrampus longispinosus Ozsvárt, Dumitrica \& Moix, 2017

paratype, PAL 2017.5.2.

Carnian, Killik Formation

Tavusçayırı Tepe, Sorgun, Turkey

Ozs17b, p. 62, fig. 7.2

No inventory number given in original publication

Monocoronella spinifera Ozsvárt, Dumitrica \& Moix, 2017

holotype, PAL 2017.3.3.

Carnian, Killik Formation

Tavusçayırı Tepe, Sorgun, Turkey

Ozs17b, p. 61, fig. 6.6

No inventory number given in original publication

Monocoronella spinifera Ozsvárt, Dumitrica \& Moix, 2017

paratype, PAL 2017.3.1.

Carnian, Killik Formation

Tavusçayırı Tepe, Sorgun, Turkey

Ozs17b, p. 61, fig. 6.4

No inventory number given in original publication

Monocoronella spinifera Ozsvárt, Dumitrica \& Moix, 2017

paratype, PAL 2017.3.2.

Carnian, Killik Formation

Tavusçayırı Tepe, Sorgun, Turkey

Ozs 17b, p. 61, fig. 6.5

No inventory number given in original publication

Monocoronella spinifera Ozsvárt, Dumitrica \& Moix, 2017

paratype, PAL 2017.3.4.

Carnian, Killik Formation

Tavusçayırı Tepe, Sorgun, Turkey

Ozs17b, p. 61, fig. 6.7

No inventory number given in original publication 
Monocoronella spinifera Ozsvárt, Dumitrica \& Moix, 2017

paratype, PAL 2017.3.5.

Carnian, Killik Formation

Tavusçayırı Tepe, Sorgun, Turkey

Ozs17b, p. 61, fig. 6.8

No inventory number given in original publication

Paramonocapnuchosphaera fusiformis Ozsvárt, Dumitrica \& Moix, 2017

holotype, PAL 2017.2.1.

Carnian, Killik Formation

Tavusçayırı Tepe, Sorgun, Turkey

Ozs17b, p. 61, fig. 6.2

No inventory number given in original publication

Paramonocapnuchosphaera fusiformis Ozsvárt,

Dumitrica \& Moix, 2017

paratype, PAL 2017.2.2.

Carnian, Killik Formation

Tavusçayırı Tepe, Sorgun, Turkey

Ozs17b, p. 61, fig. 6.3

No inventory number given in original publication

Paraweverella tenuispinosa Kozur, Moix \&

Ozsvárt, 2009

holotype, M 2009.45.1.

Carnian, Killik Formation

Tavusçayırı Tepe, Sorgun, Turkey

Koz09, p. 43, pl. 4, fig. 12

Poulpus compactus Ozsvárt, Dumitrica \& Hungerbühler in OzsvárT et al., 2017

holotype, PAL 2016.17.1.

Carnian, Killik Formation

Tavusçayırı Tepe, Sorgun, Turkey

Ozs17a, p. 141, pl. 1, fig. 2

Poulpus compactus Ozsvárt, Dumitrica \& Hungerbühler in OzsvárT et al., 2017

paratype, PAL 2016.17.2.

Carnian, Killik Formation

Tavusçayırı Tepe, Sorgun, Turkey

Ozs17a, p. 141, pl. 1, fig. 1

No inventory number given in original publication
Poulpus elegans Ozsvárt, Dumitrica \& Hungerbühler in OzsváRT et al., 2017

holotype, PAL 2016.18.1.

Carnian, Killik Formation

Tavusçayırı Tepe, Sorgun, Turkey

Ozs17a, p. 145, pl. 1, fig. 3

Poulpus kozuri Ozsvárt, Dumitrica \& Hunger-

bühler in OzsváRT et al., 2017

holotype, PAL 2016.19.1.

Carnian, Killik Formation

Tavusçayırı Tepe, Sorgun, Turkey

Ozs17a, p. 145, pl. 1, figs 4-5

Pseudostylosphaera dumitricai Ozsvárt, Moix \& Kozur, 2015

holotype, PAL 2014.128.1.

Carnian, Killik Formation

Tavusçayırı Tepe, Sorgun, Turkey

Ozs15, p. 348, fig. 6.1

Pseudostylosphaera dumitricai Ozsvárt, Moix \& Kozur, 2015

paratype, PAL 2014.128.2.

Carnian, Killik Formation

Tavusçayırı Tepe, Sorgun, Turkey

Ozs15, p. 348, fig. 6.2

Pseudostylosphaera dumitricai Ozsvárt, Moix \& Kozur, 2015

paratype, PAL 2014.128.3.

Carnian, Killik Formation

Tavusçayırı Tepe, Sorgun, Turkey

Ozs15, p. 348, fig. 6.3

Pseudostylosphaera dumitricai Ozsvárt, Moix \& Kozur, 2015

paratype, PAL 2014.128.4.

Carnian, Killik Formation

Tavusçayırı Tepe, Sorgun, Turkey

Ozs15, p. 348, fig. 6.4

Silicarmiger longospinosus Ozsvárt, Dumitrica

\& Hungerbühler in OzsvárT et al., 2017

holotype, PAL 2016.28.2.

Carnian, Killik Formation

Tavusçayırı Tepe, Sorgun, Turkey

Ozs17a, p. 158, pl. 5, fig. 9 
Silicarmiger longospinosus Ozsvárt, Dumitrica \& Hungerbühler in Ozsvárt et al., 2017 paratype, PAL 2016.28.1.

Carnian, Killik Formation

Tavusçayırı Tepe, Sorgun, Turkey

Ozs17a, p. 158, pl. 5, fig. 10

No inventory number given in original publication

Spinocapnuchosphaera hantkeni Kozur, Moix \& Ozsvárt, 2009

holotype, M 2009.31.1.

Carnian, Killik Formation

Tavusçayırı Tepe, Sorgun, Turkey

Koz09, p. 38, pl. 4, fig. 1

Spinocapnuchosphaera odoghertyi Kozur, Moix

\& Ozsvárt, 2009

holotype, M 2009.32.1.

Carnian, Killik Formation

Tavusçayırı Tepe, Sorgun, Turkey

Koz09, p. 39, pl. 3, fig. 10

Spinocapnuchosphaera szentei Kozur, Moix \&

Ozsvárt, 2009

holotype, M 2009.33.1.

Carnian, Killik Formation

Tavusçayırı Tepe, Sorgun, Turkey

Koz09, p. 39, pl. 3, fig. 12

Spinocapnuchosphaera szivesae Kozur, Moix \&

Ozsvárt, 2009

holotype, M 2009.34.1.

Carnian, Killik Formation

Tavusçayırı Tepe, Sorgun, Turkey

Koz09, p. 39, pl. 4, fig. 2

Spinocapnuchosphaera tekini tekini Kozur, Moix

\& Ozsvárt, 2009

holotype, M 2009.28.1.

Carnian, Killik Formation

Tavusçayırı Tepe, Sorgun, Turkey

Koz09, p. 38, pl. 3, fig. 5

Spinocapnuchosphaera tekini tekini Kozur, Moix

\& Ozsvárt, 2009

paratype, M 2009.28.2.

Carnian, Killik Formation

Tavusçayırı Tepe, Sorgun, Turkey

Koz09, p. 38, pl. 3, fig. 6
Spinocapnuchosphaera tekini hugluensis Kozur,

Moix \& Ozsvárt, 2009

holotype, M 2009.29.1.

Carnian, Killik Formation

Tavusçayırı Tepe, Sorgun, Turkey

Koz09, p. 38, pl. 3, fig. 7

Spinocapnuchosphaera tekini marginospinosa

Kozur, Moix \& Ozsvárt, 2009

holotype, M 2009.30.1.

Carnian, Killik Formation

Tavusçayırı Tepe, Sorgun, Turkey

Koz09, p. 38, pl. 3, fig. 8

Spinocapnuchosphaera tricuspidata Kozur, Moix \& Ozsvárt, 2009

holotype, M 2009.35.1.

Carnian, Killik Formation

Tavusçayırı Tepe, Sorgun, Turkey

Koz09, p. 40, pl. 3, fig. 9

Spinocapnuchosphaera venusta Kozur, Moix \&

Ozsvárt, 2009

holotype, M 2009.36.1.

Carnian, Killik Formation

Tavusçayırı Tepe, Sorgun, Turkey

Koz09, p. 40, pl. 3, fig. 11

Spinostylosphaera andrasi Ozsvárt, Moix \&

Kozur, 2015

holotype, PAL 2014.126.1.

Carnian, Killik Formation

Tavusçayırı Tepe, Sorgun, Turkey

Ozs15, p. 349, fig. 6.7

Spinostylosphaera andrasi Ozsvárt, Moix \&

Kozur, 2015

paratype, PAL 2014.126.2.

Carnian, Killik Formation

Tavusçayırı Tepe, Sorgun, Turkey

Ozs15, p. 349, fig. 6.9

Spinostylosphaera andrasi Ozsvárt, Moix \&

Kozur, 2015

paratype, PAL 2014.126.3.

Carnian, Killik Formation

Tavusçayırı Tepe, Sorgun, Turkey

Ozs15, p. 349, fig. 6.10 
Spinostylosphaera andrasi Ozsvárt, Moix \&

Kozur, 2015

paratype, PAL 2014.127.1.

Carnian, Killik Formation

Tavusçayırı Tepe, Sorgun, Turkey

Ozs15, p. 349, fig. 6.8

Spinostylosphaera andrasi Ozsvárt, Moix \&

Kozur, 2015

paratype, PAL 2014.127.2.

Carnian, Killik Formation

Tavusçayırı Tepe, Sorgun, Turkey

Ozs15, p. 349, fig. 6.11

Spinostylosphaera andrasi Ozsvárt, Moix \&

Kozur, 2015

paratype, PAL 2014.127.3.

Carnian, Killik Formation

Tavusçayırı Tepe, Sorgun, Turkey

Ozs15, p. 349, figs 6.12.1-2

Inventory number given erroneously in figure

caption as PAL 2014.127.3.6.

Spinostylosphaera masseti Ozsvárt, Dumitrica \&

Moix, 2017

holotype, PAL 2017.6.2.

Carnian, Killik Formation

Tavusçayırı Tepe, Sorgun, Turkey

Ozs17b, p. 65, fig. 7.4

No inventory number given in original publication

Spinostylosphaera masseti Ozsvárt, Dumitrica \& Moix, 2017

paratype, PAL 2017.6.1.

Carnian, Killik Formation

Tavusçayırı Tepe, Sorgun, Turkey

Ozs17b, p. 65, fig. 7.3

No inventory number given in original publi-

cation

Spinostylosphaera michelae Ozsvárt, Dumitrica

\& Moix, 2017

holotype, PAL 2017.8.1.

Carnian, Killik Formation

Tavusçayırı Tepe, Sorgun, Turkey

Ozs17b, p. 65, fig. 7.6

No inventory number given in original publication
Spinostylosphaera michelae Ozsvárt, Dumitrica

\& Moix, 2017

paratype, PAL 2017.8.2.

Carnian, Killik Formation

Tavusçayırı Tepe, Sorgun, Turkey

Ozs17b, p. 65, fig. 7.7

No inventory number given in original publication

Spinostylosphaera michelae Ozsvárt, Dumitrica \& Moix, 2017

paratype, PAL 2017.8.3.

Carnian, Killik Formation

Tavusçayırı Tepe, Sorgun, Turkey

Ozs17b, p. 65, fig. 7.8

No inventory number given in original publication

Spinostylosphaera michelae Ozsvárt, Dumitrica \& Moix, 2017

paratype, PAL 2017.8.4.

Carnian, Killik Formation

Tavusçayırı Tepe, Sorgun, Turkey

Ozs17b, p. 65, fig. 7.9

No inventory number given in original publication

Spinostylosphaera sengoeri Ozsvárt, Dumitrica \& Moix, 2017

holotype, PAL 2017.7.1.

Carnian, Killik Formation

Tavusçayırı Tepe, Sorgun, Turkey

Ozs17b, p. 65, fig. 7.5

No inventory number given in original publication

Spinostylosphaera vachardi Ozsvárt, Moix \&

Kozur, 2015

holotype, PAL 2014.131.1.

Carnian, Killik Formation

Tavusçayırı Tepe, Sorgun, Turkey

Ozs15, p. 349, fig. 6.15

Spinostylosphaera vachardi Ozsvárt, Moix \&

Kozur, 2015

paratype, PAL 2014.131.2.

Carnian, Killik Formation

Tavusçayırı Tepe, Sorgun, Turkey

Ozs15, p. 349, figs 6.13.1-2 
Spinostylosphaera vachardi Ozsvárt, Moix \&

Kozur, 2015

paratype, PAL 2014.131.3.

Carnian, Killik Formation

Tavusçayırı Tepe, Sorgun, Turkey

Ozs15, p. 349, fig. 6.14

Inventory number given erroneously in figure

caption as PAL 2014.131.2.

Tamonella aspinosa Ozsvárt, Dumitrica \&

Moix, 2017

holotype, PAL 2017.4.1.

Carnian, Killik Formation

Tavusçayırı Tepe, Sorgun, Turkey

Ozs17b, p. 61, fig. 6.10

No inventory number given in original publication

Tamonella aspinosa Ozsvárt, Dumitrica \&

Moix, 2017

paratype, PAL 2017.4.2.

Carnian, Killik Formation

Tavusçayırı Tepe, Sorgun, Turkey

Ozs17b, p. 61, fig. 6.11

No inventory number given in original publication

Tekinium bragini Ozsvárt, Dumitrica \&

Hungerbühler in OzsváRT et al., 2017

holotype, PAL 2016.24.1.

Carnian, Killik Formation

Tavusçayırı Tepe, Sorgun, Turkey

Ozs17a, p. 155, pl. 3, fig. 12

Tekinium bragini Ozsvárt, Dumitrica \&

Hungerbühler in Ozsvárt et al., 2017

paratype, PAL 2016.24.2.

Carnian, Killik Formation

Tavusçayırı Tepe, Sorgun, Turkey

Ozs17a, p. 155, pl. 3, fig. 11

No inventory number given in original publication

Tetracapnuchosphaera? voeroesi Kozur, Moix \& Ozsvárt, 2009

holotype, M 2009.38.1.

Carnian, Killik Formation

Tavusçayırı Tepe, Sorgun, Turkey

Koz09, p. 40, pl. 4, fig. 4
Triassobullasphaera miriae Ozsvárt, Moix \&

Kozur, 2015

holotype, PAL 2014.125.1.

Carnian, Killik Formation

Tavusçayırı Tepe, Sorgun, Turkey

Ozs15, p. 344, fig. 5.5

Triassobullasphaera miriae Ozsvárt, Moix \&

Kozur, 2015

paratype, PAL 2014.125.2.

Carnian, Killik Formation

Tavusçayırı Tepe, Sorgun, Turkey

Ozs15, p. 344, fig. 5.6

Tubospongopallium inaequispinosum Kozur,

Moix \& Ozsvárt, 2009

holotype, M 2009.46.1.

Carnian, Killik Formation

Tavusçayırı Tepe, Sorgun, Turkey

Koz09, p. 43, pl. 4, fig. 13

Weverella gracilispinosa Kozur, Moix \&

Ozsvárt, 2009

holotype, M 2009.40.1.

Carnian, Killik Formation

Tavusçayırı Tepe, Sorgun, Turkey

Koz09, p. 41, pl. 4, fig. 6

Weverella gracilispinosa Kozur, Moix \&

Ozsvárt, 2009

paratype, M 2009.40.2.

Carnian, Killik Formation

Tavusçayırı Tepe, Sorgun, Turkey

Koz09, p. 41, pl. 4, fig. 5

Weverella longispinosa longispinosa Kozur, Moix \& Ozsvárt, 2009

holotype, M 2009.41.1.

Carnian, Killik Formation

Tavusçayırı Tepe, Sorgun, Turkey

Koz09, p. 42, pl. 4, fig. 7

Weverella longispinosa curvata Kozur, Moix \&

Ozsvárt, 2009

holotype, M 2009.42.1.

Carnian, Killik Formation

Tavusçayırı Tepe, Sorgun, Turkey

Koz09, p. 42, pl. 4, fig. 8 
Weverella longispinosa subrectangularis Kozur, Moix \& Ozsvárt, 2009

holotype, M 2009.43.1.

Carnian, Killik Formation

Tavusçayırı Tepe, Sorgun, Turkey

Koz09, p. 42, pl. 4, fig. 9

Inventory number given erroneously in figure caption as M 2009.43.11.

\subsection{Triassic Gastropoda}

Hungariella coronata Szabó, 2011

holotype, M 2010.427.1.

Norian-Rhaetian, Dachstein Limestone

Formation

Remete-hegy, Budapest

Sza11, p. 43, figs 2.5-8

Hungariella coronata Szabó, 2011

paratype, M 2010.425.1.

Norian-Rhaetian, Dachstein Limestone

Formation

Remete-hegy, Budapest

Sza11, p. 43, fig. 2.12

Hungariella coronata Szabó, 2011

paratype, M 2010.428.1.

Norian-Rhaetian, Dachstein Limestone

Formation

Remete-hegy, Budapest

Sza11, p. 43, figs 2.9-11

Trochus (Mesotrochus) triadicus Kutassy, 1927 neotype, M 2010.422.1.

Norian-Rhaetian, Dachstein Limestone

Formation

Remete-hegy, Budapest

Kut27, p. 152 (pl. 5, figs 7a-c)

Callotrochus triadicus, Sza1 1

Neotype designated and figured in SzABó

2011: p. 41, figs 1.1-3, 1.7-9

Trochus (Mesotrochus) triadicus elegantula

Kutassy, 1933

holotype, M 2010.423.1.

Norian-Rhaetian, Dachstein Limestone

Formation

Remete-hegy, Budapest

Kut33, p. 236, pl. 2, fig. 26

Callotrochus triadicus, Sza 11

Type figured in SzABó 2011: figs 1.4-6

\subsection{Triassic Cephalopoda}

Epikellnerites pseudocholnokyi Vörös, 2018 holotype, PAL 2017.20.1.

Anisian, Vászoly Formation bed 9, section I, Mencshely VöA18, p. 71, pl. 9, figs 4a-b

Epikellnerites pseudocholnokyi Vörös, 2018 paratype, PAL 2017.21.1.

Anisian, Vászoly Formation

Mencshely

VöA18, p. 71, pl. 9, figs 5a-b

Epikellnerites pseudocholnokyi Vörös, 2018 paratype, PAL 2017.22.1.

Anisian, Vászoly Formation

Mencshely

VöA18, p. 71, pl. 9, figs 3a-b

Epikellnerites pseudocholnokyi Vörös, 2018 paratype, PAL 2017.48.1.

Anisian, Vászoly Formation

Mencshely

VöA18, p. 71, text-figs 31-32

Epikellnerites spinatus Vörös, 2018

holotype, PAL 2017.23.1.

Anisian, Vászoly Formation

bed 9, section I, Mencshely

VöA18, p. 72, text-fig. 33, pl. 9, figs 6a-b

Epikellnerites spinatus Vörös, 2018

paratype, PAL 2017.24.1.

Anisian, Vászoly Formation

Mencshely

VöA18, p. 72, pl. 9, fig. 7

Epikellnerites tamasi Vörös, 2018

holotype, PAL 2017.10.1.

Anisian, Vászoly Formation?

vineyards, Szentbékkálla

VöA18, p. 67, text-figs 26-27, pl. 7, figs 3a-b

Epikellnerites vaszolyensis Vörös, 2018

holotype, PAL 2017.12.1.

Anisian, Vászoly Formation

trench $\mathrm{P}-11 / \mathrm{c}$, Vászoly

VöA18, p. 69, pl. 7, figs 6a-b 
Epikellnerites vaszolyensis Vörös, 2018 paratype, M.87.6

Anisian, Vászoly Formation?

Szentbékkálla

VöA18, p. 69, text-fig. 30, pl. 8, figs 5a-b

Epikellnerites vaszolyensis Vörös, 2018

paratype, M.98.14

Anisian, Vászoly Formation

Vászoly

VöA18, p. 69, pl. 8, figs 2a-b

Epikellnerites vaszolyensis Vörös, 2018

paratype, M.98.74

Anisian, Vászoly Formation

bed 8 , section I, Mencshely

VöA18, p. 69, text-fig. 28c, pl. 8, figs 4a-b

Epikellnerites vaszolyensis Vörös, 2018

paratype, PAL 2017.13.1.

Anisian, Vászoly Formation

trench $\mathrm{P}-11 / \mathrm{c}$, Vászoly

VöA18, p. 69, text-fig. 28b, pl. 8, figs 1a-b

Epikellnerites vaszolyensis Vörös, 2018

paratype, PAL 2017.14.1.

Anisian, Vászoly Formation

trench $\mathrm{P}-11 / \mathrm{c}$, Vászoly

VöA18, p. 69, text-fig. 29, pl. 8, figs 3a-b

Epikellnerites vaszolyensis Vörös, 2018

paratype, PAL 2017.15.1.

Anisian, Vászoly Formation

bed 9, section I, Mencshely

VöA18, p. 69, pl. 8, figs 6a-b

Epikellnerites vaszolyensis Vörös, 2018

paratype, PAL 2017.16.1.

Anisian, Vászoly Formation

shaft P-XVIII, Vászoly

VöA18, p. 69, pl. 8, figs 7a-b

Epikellnerites vaszolyensis Vörös, 2018

paratype, PAL 2017.17.1.

Anisian, Vászoly Formation

Mencshely

VöA18, p. 69, pl. 8, figs 8a-b
Epikellnerites vaszolyensis Vörös, 2018

paratype, PAL 2017.18.1.

Anisian, Vászoly Formation

shaft P-XVIII, Vászoly

VöA18, p. 69, pl. 9, figs 1a-b

Epikellnerites vaszolyensis Vörös, 2018

paratype, PAL 2017.19.1.

Anisian, Vászoly Formation

bed 9, section I, Mencshely

VöA18, p. 69, pl. 9, figs 2a-b

Epikellnerites vaszolyensis Vörös, 2018

paratype, PAL 2017.47.1.

Anisian, Vászoly Formation

Mencshely

VöA18, p. 69, text-fig. 28a

Hungarites sinuosus Vörös, 2018

holotype, PAL 2017.38.1.

Anisian, Vászoly Formation

bed 6, section I, Mencshely

VöA18, p. 120, text-figs 70a, 71, pl. 34, figs 5a-b

Hungarites sinuosus Vörös, 2018

paratype, M.89.100

Anisian, Vászoly Formation

bed 16/A, trench P-11/a, Vászoly

VöA18, p. 120, text-fig. 70b, pl. 34, figs 4a-b

Hungarites sinuosus Vörös, 2018

paratype, PAL 2017.39.1.

Anisian, Vászoly Formation

Vászoly

VöA18, p. 120, pl. 35, figs 1a-b

Hungarites sinuosus Vörös, 2018

paratype, PAL 2017.40.1.

Anisian, Vászoly Formation

Section I, Mencshely

VöA18, p. 120, pl. 35, figs $2 a-b$

Hungarites sinuosus Vörös, 2018

paratype, PAL 2017.41.1.

Anisian, Vászoly Formation

Vászoly

VöA18, p. 120, pl. 35, figs 3a-b 
Hungarites szentei Vörös, 2018

holotype, PAL 2017.42.1.

Anisian, Vászoly Formation trench P-14, Vászoly

VöA18, p. 121, text-fig. 72, pl. 36, figs 1a-b

Hungarites szentei Vörös, 2018

paratype, PAL 2017.43.1.

Anisian, Vászoly Formation

trench P-14, Vászoly

VöA18, p. 121, pl. 36, figs 2a-b

Hungarites szentei Vörös, 2018

paratype, PAL 2017.44.1.

Anisian, Vászoly Formation

bed 6, Szentkirályszabadja

VöA18, p. 121, pl. 36, figs 3a-b

Hungarites szentei Vörös, 2018

paratype, PAL 2017.45.1.

Anisian, Vászoly Formation

bed 5, Szentkirályszabadja

VöA18, p. 121, pl. 36, fig. 4

Hyparpadites szaboi Vörös, 2018

holotype, PAL 2017.25.1.

Anisian, Vászoly Formation

shaft P-XVII, Vászoly

VöA18, p. 85, text-fig. 43, pl. 11, figs 4a-b

Hyparpadites szaboi Vörös, 2018

paratype, PAL 2017.26.1.

Anisian, Vászoly Formation

shaft P-XVII, Vászoly

VöA18, p. 85, pl. 11, figs 6a-b

Hyparpadites szaboi Vörös, 2018

paratype, PAL 2017.27.1.

Anisian, Vászoly Formation

bed 14, section I, Mencshely

VöA18, p. 85, pl. 11, fig. 3

Nodihungarites vinczei Vörös, 2018

holotype, M.89.81

Anisian, Vászoly Formation

bed 16/A, trench P-11/a, Vászoly

VöA18, p. 127, pl. 40, figs 1a-b
Nodihungarites vinczei Vörös, 2018

paratype, PAL 2017.46.1.

Anisian, Vászoly Formation

trench $\mathrm{P}-11 / \mathrm{a}$, Vászoly

VöA18, p. 127, pl. 38, fig. 7

Parahungarites solyensis Vörös, 2018

holotype, M.98.53

Anisian, Vászoly Formation

bed 9, Ör-hegy, Sóly

VöA18, p. 98, pl. 18, figs 8a-b

Parahungarites solyensis Vörös, 2018

paratype, M.98.208A

Anisian, Vászoly Formation

bed 6, Ör-hegy, Sóly

VöA18, p. 98, pl. 18, figs 5a-b

Parahungarites solyensis Vörös, 2018

paratype, M.98.208B

Anisian, Vászoly Formation

bed 6, Ör-hegy, Sóly

VöA18, p. 98, text-fig. 57, pl. 18, figs 9a-b

Parahungarites solyensis Vörös, 2018

paratype, M.98.212A

Anisian, Vászoly Formation

bed 6, Ör-hegy, Sóly

VöA18, p. 98, pl. 18, figs 7a-b

Parahungarites solyensis Vörös, 2018

paratype, PAL 2017.32.1.

Anisian, Vászoly Formation

trench P-17, Vászoly

VöA18, p. 98, pl. 18, figs 6a-b

Parakellnerites stuerzenbaumi Vörös, 2018 holotype, PAL 2017.30.1.

Anisian, Vászoly Formation

trench P-14, Vászoly

VöA18, p. 90, pl. 14, fig. 1

Parakellnerites stuerzenbaumi Vörös, 2018 paratype, M.89.83

Anisian, Vászoly Formation

bed 16/A, trench P-11/a, Vászoly

VöA18, p. 90, pl. 14, figs 4a-b 
Parakellnerites stuerzenbaumi Vörös, 2018 paratype, M.89.86

Anisian, Vászoly Formation bed 16/A, trench P-11/a, Vászoly

VöA18, p. 90, pl. 14, fig. 3

Parakellnerites stuerzenbaumi Vörös, 2018 paratype, PAL 2017.28.1.

Anisian, Vászoly Formation

bed 111/E, Felsőörs

VöA18, p. 90, pl. 13, figs 6a-b

Parakellnerites stuerzenbaumi Vörös, 2018 paratype, PAL 2017.29.1.

Anisian, Vászoly Formation

bed 111/F, Felsőörs

VöA18, p. 90, pl. 13, fig. 5

Parakellnerites stuerzenbaumi Vörös, 2018

paratype, PAL 2017.31.1.

Anisian, Vászoly Formation

bed 111/F, Felsőörs

VöA18, p. 90, pl. 14, figs 2a-b

Stoppaniceras budaii Vörös, 2018

holotype, M.87.42

Anisian, Vászoly Formation

bed 16/A, trench P-11/a, Vászoly

VöA18, p. 107, text-figs 60-61, pl. 27, figs 3a-b

Stoppaniceras budaii Vörös, 2018

paratype, PAL 2017.37.1.

Anisian, Vászoly Formation

bed 111, Felsőörs

VöA18, p. 107, pl. 27, figs 2a-b

Stoppaniceras hermanni Vörös, 2018

holotype, M.98.16

Anisian, Vászoly Formation

bed 16/A, trench P-11/a, Vászoly

VöA18, p. 106, pl. 26, figs 5a-b

Stoppaniceras hermanni Vörös, 2018

paratype, PAL 2017.36.1.

Anisian, Vászoly Formation

trench P-17, Vászoly

VöA18, p. 106, pl. 27, figs 1a-b
Stoppaniceras rieberi Vörös, 2018

holotype, M.98.26

Anisian, Vászoly Formation

bed 116, Felsöörs

VöA18, p. 103, pl. 25, figs 3a-b

Stoppaniceras rieberi Vörös, 2018

paratype, M.98.27

Anisian, Vászoly Formation

bed 116, Felsőörs

VöA18, p. 103, pl. 25, fig. 7

Stoppaniceras rieberi Vörös, 2018

paratype, PAL 2017.33.1.

Anisian, Vászoly Formation

bed 111/I, Felsőörs

VöA18, p. 103, pl. 25, figs 4a-b

Stoppaniceras rieberi Vörös, 2018

paratype, PAL 2017.34.1.

Anisian, Vászoly Formation

bed 112, Felsőörs

VöA18, p. 103, pl. 25, fig. 6

Stoppaniceras rieberi Vörös, 2018

paratype, PAL 2017.35.1.

Anisian, Vászoly Formation

bed 112, Felsőörs

VöA18, p. 103, pl. 25, fig. 5

\section{Jurassic types}

\subsection{Jurassic Bivalvia}

Pholadomya schafarziki Papp, 1907

holotype, M.63.3198

Callovian-Oxfordian, "limestone"

Gunib, Daghestan, Russia

Pap07, p. 155, pl. 3, figs 1, 1a-b

Pholadomya (Bucardiomya) lirata, Gal08

Type figured in Galácz \& Szente 2008: pl.

4 , figs $1-4$

Pleuromya merzbacheri Papp, 1907

holotype, M.63.3616

Callovian

Psebay, Kuban, Russia

Pap07, p. 158, pl. 4, figs 2, 2a-c

Pleuromya alduini, Gal08

Type figured in Galácz \& Szente 2008: pl.

4, figs 8-9 


\subsection{Jurassic Gastropoda}

Adeorbisina procera Szabó, 1981 paratypes, PAL 2018.34.1., PAL 2018.35.1., PAL 2018.36.1-4.

Bajocian, fissure-filling of Csókakő Limestone Formation?

Som-hegy, Bakonybél

Sza81, p. 63

Mariottia gibbosa, Con87

Anoptychia hastata Szabó, 1983

paratype, PAL 2018.37.1.

Bajocian, fissure-filling of Csókakő Limestone

Formation?

Som-hegy, Bakonybél

Sza83, p. 34

Bakonyia planapex Szabó, 1981

paratypes, PAL 2018.38.1-2., PAL 2018.39.1-

2., PAL 2018.40.1-2.

Bajocian, fissure-filling of Csókakő Limestone

Formation?

Som-hegy, Bakonybél

Sza81, p. 61

Bathrotomaria mandokii Szabó, 1980

paratype, PAL 2018.41.1.

Bajocian, fissure-filling of Csókakő Limestone

Formation?

Som-hegy, Bakonybél

Sza80, p. 61

Dimorphotectus unicarinatus Szabó, 1981

paratypes, PAL 2018.42.1-3., PAL 2018.43.3-4.

Bajocian, fissure-filling of Csókakő Limestone

Formation?

Som-hegy, Bakonybél

Sza81, p. 58

Eucycloidea galaczi Szabó, 1983

paratypes, PAL 2018.43.5., PAL 2018.45.1-3.,

PAL 2018.46.1-2., PAL 2018.47.1., PAL

2018.48.1.

Bajocian, fissure-filling of Csókakő Limestone Formation?

Som-hegy, Bakonybél

Sza83, p. 42
Eucycloidea galaczi Szabó, 1983

paratype, PAL 2018.44.1.

Bajocian, fissure-filling of Csókakő Limestone

Formation?

Som-hegy, Bakonybél

Sza83, p. 42, pl. 3 , fig. 8

Laevitomaria danii Szabó, 2009

holotype, M 2008.542.1.

Pliensbachian, Hierlatz Limestone Formation

Fenyveskút, Lókút

Sza09, p. 49, figs 43a-c

Leptomaria sombegyensis Szabó, 1980

paratype, PAL 2018.49.1.

Bajocian, fissure-filling of Csókakő Limestone

Formation?

Som-hegy, Bakonybél

Sza80, p. 59, pl. 3, fig. 2

Trochotomaria sombegyensis, Con 87

Leptomaria somhegyensis Szabó, 1980

paratypes, PAL 2018.50.1-4.

Bajocian, fissure-filling of Csókakő Limestone

Formation?

Som-hegy, Bakonybél

Sza80, p. 59

Trochotomaria sombegyensis, Con 87

Neritopsis (Neritopsis) spinigera Szabó, 1982

paratypes, PAL 2018.51.1., PAL 2018.52.1.,

PAL 2018.54.1-6., PAL 2018.58.1.

Bajocian, fissure-filling of Csókakő Limestone Formation?

Som-hegy, Bakonybél

Sza82, p. 19

Neritopsis (Neritopsis) spinigera Szabó, 1982 paratypes, PAL 2018.53.1-17.

Bajocian, fissure-filling of Csókakő Limestone Formation?

Som-hegy, Bakonybél

Sza82, p. 19, pl. 1, fig. 12

Neritopsis (Neritopsis) spinigera Szabó, 1982 paratype, PAL 2018.55.1.

Bajocian, fissure-filling of Csókakő Limestone

Formation?

Som-hegy, Bakonybél

Sza82, p. 19, pl. 1, figs 15-16 
Neritopsis (Neritopsis) spinigera Szabó, 1982 paratype, PAL 2018.56.1.

Bajocian, fissure-filling of Csókakő Limestone Formation?

Som-hegy, Bakonybél

Sza82, p. 19, pl. 1, figs 13-14

Neritopsis (Neritopsis) spinigera Szabó, 1982 paratype, PAL 2018.57.1.

Bajocian, fissure-filling of Csókakő Limestone

Formation?

Som-hegy, Bakonybél

Sza82, p. 19, pl. 1, figs 17-18

Ochetochilus piceus Szabó, 1983

paratypes, PAL 2018.59.1-2.

Bajocian, fissure-filling of Csókakő Limestone

Formation?

Som-hegy, Bakonybél

Sza83, p. 43, pl. 3, figs 1-3

Telleria (Telleria) picea, Con87

Pietteia trispinigera Szabó, 1983

paratypes, PAL 2018.60.1-2., PAL 2018.61.1-2.

Bajocian, fissure-filling of Csókakő Limestone

Formation?

Som-hegy, Bakonybél

Sza83, p. 40

Pietteia (Trietteia) trispinigera, Con 87

Procerithium? (Cosmocerithium?) angulocos-

tatum Szabó, 1983

paratypes, PAL 2018.62.1-2.

Bajocian, fissure-filling of Csókakő Limestone

Formation?

Som-hegy, Bakonybél

Sza83, p. 39

Diatrypesis angulocostatum, Con87

Proconulus epuliformis Szabó, 1981

paratype, PAL 2018.63.1.

Bajocian, fissure-filling of Csókakő Limestone

Formation?

Som-hegy, Bakonybél

Sza81, p. 56, pl. 1, figs 7-8

Type figured in Conti \& Szabó 1987: fig. 1e
Proconulus epuliformis Szabó, 1981

paratypes, PAL 2018.64.1-13., PAL 2018.65.1., PAL 2018.66.1-11., PAL 2018.67.1-7., PAL

2018.68.1-2., PAL 2018.69.1-4.

Bajocian, fissure-filling of Csókakő Limestone

Formation?

Som-hegy, Bakonybél

Sza81, p. 56

Proconulus rimosus Szabó, 1981

paratypes, PAL 2018.70.1-2., PAL 2018.71.1-

4., PAL 2018.72.1., PAL 2018.73.1-13., PAL

2018.74.1., PAL 2018.75.1., PAL 2018.76.1-11., PAL 2018.79.1-3.

Bajocian, fissure-filling of Csókakő Limestone

Formation?

Som-hegy, Bakonybél

Sza81, p. 57

Proconulus baldensis, Con 87

Proconulus rimosus Szabó, 1981

paratypes, PAL 2018.77.1-6.

Bajocian, fissure-filling of Csókakő Limestone

Formation?

Som-hegy, Bakonybél

Sza81, p. 57

Proconulus baldensis, Con 87

One of the paratypes figured in ConTi $\&$

SzABó 1987: fig. 2

Proconulus rimosus Szabó, 1981

paratype, PAL 2018.78.1.

Bajocian, fissure-filling of Csókakő Limestone

Formation?

Som-hegy, Bakonybél

Sza81, p. 57

Proconulus baldensis, Con 87

Type figured in Conti \& Szabó 1987: fig. 2

Pyrgotrochus? problematicus Szabó, 1980

paratype, PAL 2018.80.1.

Bajocian, fissure-filling of Csókakő Limestone

Formation?

Som-hegy, Bakonybél

Sza80, p. 63

Laevitomaria problematica, Con 87

Type figured under inventory number

INV.2012.15.1 in GATTO et al. 2015: fig. $2 c_{1-3}$ 
Pyrgotrochus? problematicus Szabó, 1980

paratype, PAL 2018.80.2.

Bajocian, fissure-filling of Csókakő Limestone

Formation?

Som-hegy, Bakonybél

Sza80, p. 63

Laevitomaria problematica, Con 87

Type figured under inventory number

INV.2012.15.2 in GATTO et al. 2015: fig. $2 \mathrm{~d}_{1-3}$

Pyrgotrochus? problematicus Szabó, 1980

paratype, PAL 2018.80.3.

Bajocian, fissure-filling of Csókakő Limestone

Formation?

Som-hegy, Bakonybél

Sza80, p. 63

Laevitomaria problematica, Con 87

Type figured under inventory number

INV.2012.15.3 in GATTO et al. 2015: fig. 2b

Pyrgotrochus? problematicus Szabó, 1980

paratypes, PAL 2018.80.4-7., PAL 2018.81.1-

6., PAL 2018.82.1., PAL 2018.83.1.

Bajocian, fissure-filling of Csókakő Limestone

Formation?

Som-hegy, Bakonybél

Sza80, p. 63

Laevitomaria problematica, Con 87

Trochopsidea kondai Szabó, 1981

paratypes, PAL 2018.84.1., PAL 2018.85.1-4.,

PAL 2018.86.1-11., PAL 2018.87.1-5.

Bajocian, fissure-filling of Csókakő Limestone

Formation?

Som-hegy, Bakonybél

Sza81, p. 62

Ventricaria? vesicula Szabó, 1983

paratypes, PAL 2018.88.1-2.

Bajocian, fissure-filling of Csókakő Limestone

Formation?

Som-hegy, Bakonybél

Sza83, p. 30, pl. 1, fig. 10

Ventricaria? vesicula Szabó, 1983

paratypes, PAL 2018.89.1-2.

Bajocian, fissure-filling of Csókakő Limestone

Formation?

Som-hegy, Bakonybél

Sza83, p. 30, pl. 1, figs 8-9
Zircia zircensis Szabó, 1981

paratypes, PAL 2018.90.1-2., PAL 2018.91.1.,

PAL 2018.92.1., PAL 2018.93.1.

Bajocian, fissure-filling of Csókakő Limestone

Formation?

Som-hegy, Bakonybél

Sza81, p. 64

\subsection{Jurassic Cephalopoda}

Perisphinctes daghestanicus Papp, 1907

holotype, M.63.3304A

Bajocian, "lower dark shale"

Gunib, Daghestan, Russia

Pap07, p. 166, pl. 7, figs 3, 3a

Prorsisphinctes loczyi, Gal08

Type figured in Galácz \& Szente 2008: pl.

3 , fig. 7 , specimen found in collection in 2008

Perisphinctes lóczyi Papp, 1907

lectotype, M.63.3301A

Bajocian, "lower dark shale"

Gunib, Daghestan, Russia

Pap07, p. 165, pl. 7, fig. 1

Prorsisphinctes loczyi, Gal08

Lectotype designated and figured in GALÁCZ

\& SZENTE 2008: p. 114, pl. 3, figs 1-2

Perisphinctes lóczyi Papp, 1907

paralectotype, M.63.3301B

Bajocian, "lower dark shale"

Gunib, Daghestan, Russia

Pap07, p. 165, pl. 7, figs 2, 2a-b

Prorsisphinctes loczyi, Gal08

Paralectotype No. 1 figured in GALÁCZ \&

SZENTE 2008: pl. 3, figs 5-6

Perisphinctes lóczyi Papp, 1907

paralectotype, M.63.3208

Bajocian, "lower dark shale"

Gunib, Daghestan, Russia

Pap07, p. 165

Prorsisphinctes loczyi, Gal08

Counterpart of paralectotype No. 1, specimen found in collection in 2008 
Perisphinctes lóczyi Papp, 1907

paralectotype, M.63.3304A

Bajocian, "lower dark shale"

Gunib, Daghestan, Russia

Pap07, p. 165

Prorsisphinctes loczyi, Gal08

Paralectotype No. 2 figured in GALÁcz \&

SZENTE 2008: pl. 3, fig. 7, specimen found in collection in 2008

Perisphinctes lóczyi Papp, 1907

paralectotype, M.63.3304B

Bajocian, "lower dark shale"

Gunib, Daghestan, Russia

Pap07, p. 165

Prorsisphinctes loczyi, Gal08

Paralectotype No. 3, specimen found in collection in 2008

Simoceras agostyani Főzy \& Scherzinger, 2013

holotype, PAL 2013.26.1.

Tithonian, Szentivánhegy Limestone

Formation

bed 12, section I, Szomód

Főz13, p. 254, pl. 27, figs 1a-b

Original species name emended here as agostyanense

Simoceras szentei Főzy \& Scherzinger, 2011

holotype, PAL 2011.4.1.

Tithonian, Szentivánhegy Limestone

Formation

bed 5, Paprét-árok, Süttő

Főz11, p. 124, figs 5.1, 6.2

Stephanoceras liechtensteinii Papp, 1907

holotype, M.63.3610

Bajocian, "dark ferruginous limestone boulder"

Fiagdon Creek, west of Vladikavkaz, Russia Pap07, p. 163, pl. 6, fig. 4

Type figured in GaLÁCZ \& SZENTE 2008: pl.

2 , figs $5-6$

Virgatosimoceras dunaii Scherzinger, Főzy \&

Parent, 2010

holotype, M.92.749

Tithonian, Pálihálás Limestone Formation

bed 47, Lókúti-domb, Lókút

ScA10, p. 201, figs $4.1 \mathrm{a}-\mathrm{b}$

\subsection{Jurassic Brachiopoda}

Apringia barnabasi Vörös, 2009

holotype, 2007.121.1.

Pliensbachian, Hierlatz Limestone Formation bed 26, Kericser section, Lókút VöA09, p. 45, pl. 1, figs 6a-c

Apringia barnabasi Vörös, 2009 paratype, 2007.122.1.

Pliensbachian, Hierlatz Limestone Formation bed 16, Kericser section, Lókút VöA09, p. 45, pl. 1, figs 7a-c

Cirpa alkayae Vörös, 2014

holotype, PAL 2014.137.1.

Pliensbachian, Bayırköy Formation

Beytepe (Lodumlu), Ankara area, Turkey

VöA14b, p. 19, figs 17-20

Cirpa alkayae Vörös, 2014

paratype, PAL 2014.138.1.

Pliensbachian, Bayırköy Formation

Beytepe (Lodumlu), Ankara area, Turkey

VöA14b, p. 19, fig. 23

Fenyveskutella pseudoubligi Vörös, 2009

holotype, 2007.229.1.

Pliensbachian, Tűzkövesárok Limestone

Formation

basal layer, Fg-I, Gombáspuszta, Szentgál

VöA09, p. 90, pl. 10, figs 9a-c

Fenyveskutella pseudoubligi Vörös, 2009

paratype, 2007.228.1.

Pliensbachian, Hierlatz Limestone Formation

Kericser section, Lókút

VöA09, p. 90, pl. 10, figs 8a-c

Fenyveskutella theresiae Vörös, 2009

holotype, 2007.231.1.

Pliensbachian, Hierlatz Limestone Formation grab sample É, Fenyveskút, Lókút

VöA09, p. 91, pl. 10, figs 11a-c

Fenyveskutella vighi Vörös, 2009

holotype, 2007.221.1.

Pliensbachian, Hierlatz Limestone Formation grab sample p1, Fenyveskút, Lókút

VöA09, p. 88, pl. 10, figs 1a-c 
Fenyveskutella vighi Vörös, 2009

paratype, 2007.222.1.

Pliensbachian, Hierlatz Limestone Formation grab sample É, Fenyveskút, Lókút

VöA09, p. 88, pl. 10, figs 2a-c

Karadagella szentei Vörös, 2013

holotype, PAL 2013.1.1.

Oxfordian, Pálihálás Limestone Formation?

Velka Skala, Kétágú-hegy, Kesztölc

VöA13, p. 384, pl. 1, figs 6a-c

Karadagella szentei Vörös, 2013

paratype, PAL 2013.2.1.

Oxfordian, Pálihálás Limestone Formation?

Velka Skala, Kétágú-hegy, Kesztölc

VöA13, p. 384, text-fig. 4

Paronarhynchia estherae Vörös, 2009

holotype, 2007.237.1.

Pliensbachian, Hierlatz Limestone Formation grab sample p2, Fenyveskút, Lókút

VöA09, p. 101, pl. 11, figs 1a-c

Paronarbynchia estherae Vörös, 2009

paratype, 2007.239.1.

Pliensbachian, Hierlatz Limestone Formation grab sample p1, Fenyveskút, Lókút

VöA09, p. 101, pl. 11, figs 3a-c

Prionorhynchia? catharinae Vörös, 2009

holotype, 2007.192.1.

Pliensbachian, Hierlatz Limestone Formation grab sample D, Fenyveskút, Lókút

VöA09, p. 70, pl. 8, figs 6a-c

Rhapidothyris lokutica Vörös, 2009

holotype, M 2008.391.1.

Pliensbachian, Hierlatz Limestone Formation bed 8, Kericser section, Lókút

VöA09, p. 145, pl. 16, figs 4a-c

Rhapidothyris lokutica Vörös, 2009

paratype, M 2008.390.1.

Pliensbachian, Isztimér Limestone Formation bed 3, Büdöskút, Gyulafirátót, Veszprém

VöA09, p. 145, pl. 16, figs 3a-c

\section{Cretaceous types}

\subsection{Cretaceous Cephalopoda}

Ancyloceras capellini Matheron, 1880

holotype, M.62.3

Barremian

Clumanc, France

Mat80, pl. C-24, figs 1a-b

Heteroceras? capellini, Kle07

Specimen previously in permanent exhibition

of the museum, returned to collection in 2013

?Chigaroceras szomodi Szives \& Főzy, 2013

holotype, PAL 2013.29.1.

Berriasian, Szentivánhegy Limestone

Formation

bed 10, section I, Szomód

Szi13, p. 319, pl. 6, figs 1a-b

Original species name emended here as $s z 0$ modense

\subsection{Cretaceous Brachiopoda}

Sphenope bifida Vörös, 2014

holotype, PAL 2013.27.1.

Berriasian, Szentivánhegy Limestone

Formation

bed 41, Szilas-árok, Borzavár

VöA14a, p. 9, figs 26-28

Sphenope bifida Vörös, 2014

paratype, PAL 2013.28.1.

Berriasian, Szentivánhegy Limestone

Formation

bed 41, Szilas-árok, Borzavár

VöA14a, p. 9, fig. 36

\subsection{Cretaceous Crinoidea}

Apsidocrinus doreckae Konieczyński, Pisera \& Fözy, 2016

holotype, PAL 2016.8.1.

Barremian, Bersek Marl Formation

bed 252, Bersek-hegy, Lábatlan

KPF16, p. 162, figs 41-m 
Apsidocrinus doreckae Konieczyński, Pisera \&

Fözy, 2016

paratype, PAL 2016.9.1.

Barremian, Bersek Marl Formation

bed 252, Bersek-hegy, Lábatlan

KPF16, p. 162, fig. 4n

Phyllocrinus bersekensis Konieczyński, Pisera \&

Fözy, 2016

holotype, PAL 2016.6.1.

Barremian, Bersek Marl Formation

bed 257, Bersek-hegy, Lábatlan

KPF16, p. 157, figs 3k-1

Phyllocrinus bersekensis Konieczyński, Pisera \&

Fözy, 2016

paratype, PAL 2016.7.1.

Barremian, Bersek Marl Formation

bed 258, Bersek-hegy, Lábatlan

KPF16, p. 157, fig. 3i

\subsection{Cretaceous Echinoidea}

Absurdaster hungaricus Kroh, Lukeneder \&

Gallemí, 2014

holotype, PAL 2013.30.1.

Hauterivian, Bersek Marl Formation

bed 221, Bersek-hegy, Lábatlan

Kro14, p. 244, figs 5.3, 6.3a-b, 7.1a-c, 8.1a-b

Absurdaster hungaricus Kroh, Lukeneder \&

Gallemí, 2014

paratype, PAL 2013.31.1.

Hauterivian, Bersek Marl Formation

bed 226, Bersek-hegy, Lábatlan

Kro14, p. 244, figs 7.2, 8.2

Absurdaster hungaricus Kroh, Lukeneder \&

Gallemí, 2014

paratype, PAL 2013.32.1.

Hauterivian, Bersek Marl Formation

bed 228, Bersek-hegy, Lábatlan

Kro14, p. 244, figs 5.4, 6.4a-b, 7.3a-c, 8.3

Absurdaster hungaricus Kroh, Lukeneder \&

Gallemí, 2014

paratype, PAL 2013.33.1.

Hauterivian, Bersek Marl Formation

bed 233, Bersek-hegy, Lábatlan

Kro14, p. 244, figs 7.4a-b, 9a-c
4. Eocene types

\subsection{Eocene Foraminiferida}

Globigerina applanata Hantken, 1883

lectotype, M.72.553

Priabonian, "marl"

l'Escarène (Scarena), France

Han83a (Han83b), p. 132 (11), pl. 2, figs 7a-c

Turborotalia applanata, Szt73

Lectotype designated and figured in SzTRÁ Kos 1973: p. 224, pl. 1, figs 3a-c. Type found in collection in 2009

Globigerina applanata Hantken, 1883

paralectotype, M.72.554

Priabonian, "marl"

l'Escarène (Scarena), France

Han83a (Han83b), p. 132 (11)

Turborotalia applanata, Szt73

Type figured in SzTrÁkos 1973: pl. 1, figs

$4 a-c$. Type found in collection in 2009

Globigerina applanata Hantken, 1883

paralectotype, M.72.555

Priabonian, "marl"

l'Escarène (Scarena), France

Han83a (Han83b), p. 132 (11)

Turborotalia applanata, Szt73

Type figured in SzTRÁ Kos 1973: pl. 1, figs

5a-c. Type found in collection in 2009

Globigerina applanata Hantken, 1883

paralectotypes, M.72.556, M.72.557, M.72.558

Priabonian, "marl"

l'Escarène (Scarena), France

Han83a (Han83b), p. 132 (11)

Turborotalia applanata, Szt73

Types found in collection in 2009

Globigerina globosa Hantken, 1883

neotype, M.72.548

Priabonian, "marl"

l'Escarène (Scarena), France

Han83a (Han83b), p. 132 (11) (pl. 2, figs 3a-b)

Globigerinatheca globosa, Szt73

Neotype designated and figured in SzTRÁ Kos

1973: p. 228, pl. 2, figs 1a-c. Type found in

collection in 2009 


\subsection{Eocene Polyplacophora}

Lepidochitona viciani Dell'Angelo, Sosso, Kroh \& Dulai, 2015

paratype, PAL 2013.34.1.

Lutetian-Bartonian, Kincses Formation

roadcut at the vineries, Gánt

Del15, p. 363

Head valve

Lepidochitona viciani Dell'Angelo, Sosso, Kroh \& Dulai, 2015

paratype, PAL 2013.35.1.

Lutetian-Bartonian, Kincses Formation

roadcut at the vineries, Gánt

Del15, p. 363

Intermediate valve

Lepidochitona viciani Dell'Angelo, Sosso, Kroh \& Dulai, 2015

paratype, PAL 2013.36.1

Lutetian-Bartonian, Kincses Formation

roadcut at the vineries, Gánt

Del15, p. 363, figs 5g-i, 6d

Tail valve

\subsection{Eocene Brachiopoda}

Magellania hantkeni Meznerics, 1944

neotype, M 2009.568.1.

Lutetian, Szőc Limestone Formation

Öreg-hegy quarry, Csojjányos-völgy, Kislőd

Mez44, p. 46 (pl. 3, figs 13-16, pl. 5, figs

21-23)

Meznericsia hantkeni, Bit11

Neotype designated and figured in BITNER et al. 2011: p. 121, figs 6g-i

Rugia zagorseki Dulai, 2011

holotype, M 2010.281.1.

Priabonian, Perwang Formation

2377.5 m, borehole Perwang-1, Austria

Dul11a, p. 302, figs 6e-f

Rugia zagorseki Dulai, 2011

paratype, M 2010.278.1.

Priabonian, Perwang Formation

3194 m, borehole Helmberg-1, Austria

Dul11a, p. 302, fig. 6a
Rugia zagorseki Dulai, 2011

paratype, M 2010.279.1.

Priabonian, Perwang Formation

3194 m, borehole Helmberg-1, Austria

Dul11a, p. 302, figs 6b-c

Rugia zagorseki Dulai, 2011

paratype, M 2010.280.1.

Priabonian, Perwang Formation

2083.5 m, borehole Perwang-1, Austria

Dul11a, p. 302, fig. 6d

Terebratulina johansenae Dulai, 2011

holotype, M 2010.275.1.

Priabonian, Perwang Formation

2073 m, borehole Perwang-1, Austria

Dul11a, p. 300, fig. $5 f$

Terebratulina johansenae Dulai, 2011

paratype, M 2010.270.1.

Priabonian, Perwang Formation

$2054.5 \mathrm{~m}$, borehole Perwang-1, Austria

Dul11a, p. 300, fig. 5a

Terebratulina johansenae Dulai, 2011

paratype, M 2010.271.1.

Priabonian, Perwang Formation

2054.5 m, borehole Perwang-1, Austria

Dul11a, p. 300, fig. 5b

Terebratulina johansenae Dulai, 2011

paratype, M 2010.272.1.

Priabonian, Perwang Formation

2073 m, borehole Perwang-1, Austria

Dul11a, p. 300, fig. 5c

Terebratulina johansenae Dulai, 2011 paratype, M 2010.273.1.

Priabonian, Perwang Formation

2073 m, borehole Perwang-1, Austria

Dul11a, p. 300, fig. 5d

Terebratulina johansenae Dulai, 2011 paratype, M 2010.274.1.

Priabonian, Perwang Formation

2073 m, borehole Perwang-1, Austria

Dul11a, p. 300, fig. 5e 
Terebratulina johansenae Dulai, 2011

paratype, M 2010.276.1.

Priabonian, Perwang Formation

$3190 \mathrm{~m}$, borehole Helmberg-1, Austria

Dul11a, p. 300, fig. $5 g$

Terebratulina johansenae Dulai, 2011

paratype, M 2010.277.1.

Priabonian, Perwang Formation

2054.5 m, borehole Perwang-1, Austria

Dul11a, p. 300, fig. 5h

\section{Oligocene types}

\subsection{Oligocene Gastropoda}

Cypraeorbis hungarica Schilder, 1932

neotype, M.63.3093

Egerian, Eger Formation

Wind brickyard, Eger

ScF32, p. 261

Specimen figured in BÁLDI 1973 (pl. 34, fig.

4) as Zonaria globosa. Neotype designated in

KovÁCs \& Vicián 2016: p. 236

Dorsanum strigoniense Kovács \& Vicián, 2016 holotype, PAL 2016.1.1.

Egerian, Törökbálint Formation

Szentgyörgymező, Esztergom

Kov16, p. 240, pl. 4, figs 11-13

Dorsanum strigoniense Kovács \& Vicián, 2016

paratype, PAL 2016.2.1.

Egerian, Törökbálint Formation

Szentgyörgymező, Esztergom

Kov16, p. 240, pl. 4, figs 14-15

Dorsanum strigoniense Kovács \& Vicián, 2016

paratype, PAL 2016.3.1.

Egerian, Törökbálint Formation

Szentgyörgymező, Esztergom

Kov16, p. 240, pl. 4, fig. 16

Dorsanum strigoniense Kovács \& Vicián, 2016

paratypes, PAL 2016.4.1., PAL 2016.5.1.

Egerian, Törökbálint Formation

Szentgyörgymező, Esztergom

Kov16, p. 240
Merica krocki Kovács \& Vicián, 2016

holotype, PAL 2016.13.1.

Egerian, Törökbálint Formation

Szentgyörgymezö, Esztergom

Kov16, p. 241, pl. 5, figs 1-2

Merica krocki Kovács \& Vicián, 2016

paratype, PAL 2016.14.1.

Egerian, Törökbálint Formation

Szentgyörgymező, Esztergom

Kov16, p. 241, pl. 5, figs 5-6

Pugilina katalinae Kovács \& Vicián, 2016 holotype, PAL 2016.10.1.

Egerian, Törökbálint Formation

Szentgyörgymezö, Esztergom

Kov16, p. 236, pl. 3, figs 2-3

Pugilina katalinae Kovács \& Vicián, 2016 paratype, PAL 2016.11.1.

Egerian, Törökbálint Formation

Szentgyörgymezö, Esztergom

Kov16, p. 236, pl. 3, figs 4-5

Pugilina katalinae Kovács \& Vicián, 2016 paratype, PAL 2016.12.1.

Egerian, Törökbálint Formation

Szentgyörgymezö, Esztergom

Kov16, p. 236, pl. 3, fig. 1

Sveltia nemethi Kovács \& Vicián, 2016

holotype, PAL 2016.15.1.

Egerian, Törökbálint Formation

Szentgyörgymezö, Esztergom

Kov16, p. 240, pl. 4, figs 25-26

\subsection{Oligocene Decapoda}

Calappa tridentata Beurlen, 1939

syntype, M.59.4676

Kiscellian, Kiscell Clay Formation

Újlaki brickyard, Kiscell, Budapest

Beu39, p. 150, text-fig. 5

Calappilia tridentata, Hyž14 
Calappa tridentata Beurlen, 1939

syntypes, M.59.4679

Kiscellian, Kiscell Clay Formation

Újlaki brickyard, Kiscell, Budapest

Beu39, p. 150, text-fig. 5

Calappilia tridentata, Hyž14

One of the syntypes figured in HYžNÝ 2016: fig. $8 \mathrm{~b}$

Calappa tridentata Beurlen, 1939

syntypes, M.59.4681

Kiscellian, Kiscell Clay Formation

Újlaki brickyard, Kiscell, Budapest

Beu39, p. 150, text-fig. 5, pl. 7, fig. 9

Calappilia tridentata, Hyž14

One of the syntypes figured in Busulini et al. 2014: pl. 3, fig. 8

Calappa tridentata Beurlen, 1939

syntype, M.60.758

Kiscellian, Kiscell Clay Formation

Újlaki brickyard, Kiscell, Budapest

Beu39, p. 150, text-fig. 5, pl. 7, fig. 10

Calappilia tridentata, Hyž14

Callianassa brevimanus Beurlen, 1939

lectotype, M.59.4684A

Kiscellian, Kiscell Clay Formation

Újlaki brickyard, Kiscell, Budapest

Beu39, p. 142, text-fig. 2

Lepidophthalmus crateriferus, Hyž 14

Lectotype designated and figured in HYŽNÝ

\& Dular 2014: p. 952, figs 2c $c_{1-3}$. Lectotype of Callianassa brevimanus selected to be the simultaneous neotype of Callianassa craterifera, which action makes $C$. brevimanus an objective junior synonym of $C$. craterifera

Callianassa brevimanus Beurlen, 1939

paralectotypes, M.59.4683

Kiscellian, Kiscell Clay Formation

Újlaki brickyard, Kiscell, Budapest

Beu39, p. 142, text-fig. 2, pl. 7, figs 5, 6

Lepidophthalmus crateriferus, Hyž 14

One of the paralectotypes figured in HYžNÝ \&

Dulai 2014: fig. 2e
Callianassa brevimanus Beurlen, 1939

paralectotype, M.59.4684B

Kiscellian, Kiscell Clay Formation

Újlaki brickyard, Kiscell, Budapest

Beu39, p. 142, text-fig. 2

Lepidophthalmus crateriferus, Hyž14

Type figured in HyžNÝ \& DUlaI 2014: fig. 2a

Callianassa brevimanus Beurlen, 1939

paralectotypes, M.59.4685

Kiscellian, Kiscell Clay Formation

Újlaki brickyard, Kiscell, Budapest

Beu39, p. 142, text-fig. 2

Lepidophthalmus crateriferus, Hyž14

Callianassa brevimanus Beurlen, 1939

paralectotypes, M.59.4690

Kiscellian, Kiscell Clay Formation

Újlaki brickyard, Kiscell, Budapest

Beu39, p. 142, text-fig. 2

Lepidophthalmus crateriferus, Hyž 14

Types originally treated as Callianassa nuda.

One of the paralectotypes figured in HYžNÝ \&

DULAI 2014: fig. 2b

Callianassa craterifera Lörenthey in

LÖRENTHEY \& BEURLEN, 1929

neotype, M.59.4684A

Kiscellian, Kiscell Clay Formation

Újlaki brickyard, Kiscell, Budapest

Lör29, p. 61 (pl. 2, figs 12a-c)

Lepidophthalmus crateriferus, Hyž 14

Genus name in original publication written as Calianassa. Neotype designated and figured in HYŽNÝ \& DULAI 2014: p. 952, figs $2 c_{1-3}$

Callianassa nuda Beurlen, 1939

paralectotype, M.59.4682

Kiscellian, Kiscell Clay Formation

Újlaki brickyard, Kiscell, Budapest

Beu39, p. 144, text-fig. 3

Ctenocheles rupeliensis, Hyž 14

Type figured in HyžNÝ \& Dulai 2014: fig. 5d

Callianassa nuda Beurlen, 1939

paralectotype, M.59.4686

Kiscellian, Kiscell Clay Formation

Újlaki brickyard, Kiscell, Budapest

Beu39, p. 144, text-fig. 3

Ctenocheles rupeliensis, Hyž14 
Callianassa nuda Beurlen, 1939

paralectotypes, M.59.4689

Kiscellian, Kiscell Clay Formation

Újlaki brickyard, Kiscell, Budapest

Beu39, p. 144, text-fig. 3

Ctenocheles rupeliensis, Hyž 14

One of the paralectotypes figured in HyžNÝ \&

DULAI 2014: fig. 5b, inventory number given

erroneously in figure caption as M.59.4869

Callianassa nuda Beurlen, 1939

paralectotype, M.59.4691

Kiscellian, Kiscell Clay Formation

Újlaki brickyard, Kiscell, Budapest

Beu39, p. 144, text-fig. 3

Ctenocheles rupeliensis, Hyž14

Type figured in HYŽNÝ \& DULAI 2014: fig. 5c. Type figured also in HYŽNÝ \& KLOMPMAKER 2015: fig. $9 \mathrm{~h}$

Homarus hungaricus Tshudy, Hyžný, Dulai \&

Jagt, 2018

holotype, PAL 2015.1.1.

Egerian, Mány Formation

401.2-406.7 m, borehole Mány-14, Mány

Tsh18, p. 171, figs 2-3

Plagiolophus sulcatus Beurlen, 1939

holotype, M.59.4692

Kiscellian, Kiscell Clay Formation

Újlaki brickyard, Kiscell, Budapest

Beu39, p. 155, pl. 7, fig. 11

Glyphithyreus sulcatus, Hyž14

Thaumastocheles rupeliensis Beurlen, 1939

lectotype, M.59.4694A

Kiscellian, Kiscell Clay Formation

Újlaki brickyard, Kiscell, Budapest

Beu39, p. 137, text-fig. 1

Ctenocheles rupeliensis, Hyž 14

Lectotype designated and figured in HYŽNÝ \& Dulai 2014: p. 956, fig. 4b. Type refigured in HYŽNÝ 2016: fig. 8a. Inventory number given erroneously in figure captions of both publications as M.59.4696A
Thaumastocheles rupeliensis Beurlen, 1939

paralectotypes, M.59.4693, M.59.4697,

M.59.4705, M.59.4707, M.59.4708, M.59.4712

Kiscellian, Kiscell Clay Formation

Újlaki brickyard, Kiscell, Budapest

Beu39, p. 137, text-fig. 1

Ctenocheles rupeliensis, Hyž 14

Thaumastocheles rupeliensis Beurlen, 1939

paralectotype, M.59.4694B

Kiscellian, Kiscell Clay Formation

Újlaki brickyard, Kiscell, Budapest

Beu39, p. 137, text-fig. 1

Ctenocheles rupeliensis, Hyž14

Type figured in HYŽNÝ \& DULAI 2014: fig. 6c

Thaumastocheles rupeliensis Beurlen, 1939

paralectotype, M.59.4696

Kiscellian, Kiscell Clay Formation

Újlaki brickyard, Kiscell, Budapest

Beu39, p. 137, text-fig. 1, pl. 7, fig. 2

Ctenocheles rupeliensis, Hyž14

Type figured in HYŽNÝ \& DULAI 2014: fig. 4d.

Type refigured in HYŽNÝ 2016: fig. 8c

Thaumastocheles rupeliensis Beurlen, 1939

paralectotypes, M.59.4700

Kiscellian, Kiscell Clay Formation

Újlaki brickyard, Kiscell, Budapest

Beu39, p. 137, text-fig. 1

Ctenocheles rupeliensis, Hyž14

One of the paralectotypes figured in HYŽNÝ \&

Dulai 2014: fig. 5a

Thaumastocheles rupeliensis Beurlen, 1939

paralectotypes, M.59.4701

Kiscellian, Kiscell Clay Formation

Újlaki brickyard, Kiscell, Budapest

Beu39, p. 137, text-fig. 1, pl. 7, fig. 1

Ctenocheles rupeliensis, Hyž14

Thaumastocheles rupeliensis Beurlen, 1939

paralectotypes, M.59.4703

Kiscellian, Kiscell Clay Formation

Újlaki brickyard, Kiscell, Budapest

Beu39, p. 137, text-fig. 1

Ctenocheles rupeliensis, Hyž 14

Types figured in HYŽNÝ \& DULAI 2014: fig. 4c 
Thaumastocheles rupeliensis Beurlen, 1939

paralectotype, M.59.4704

Kiscellian, Kiscell Clay Formation

Újlaki brickyard, Kiscell, Budapest

Beu39, p. 137, text-fig. 1

Ctenocheles rupeliensis, Hyž14

Type figured in HYžNÝ \& DUlaI 2014: fig. 4e

Thaumastocheles rupeliensis Beurlen, 1939

paralectotypes, M.59.4706

Kiscellian, Kiscell Clay Formation

Újlaki brickyard, Kiscell, Budapest

Beu39, p. 137, text-fig. 1

Ctenocheles rupeliensis, Hyž14

Types figured in HYŽNÝ \& DULAI 2014: fig. 6a

Thaumastocheles rupeliensis Beurlen, 1939

paralectotype, M.59.4709

Kiscellian, Kiscell Clay Formation

Újlaki brickyard, Kiscell, Budapest

Beu39, p. 137, text-fig. 1

Ctenocheles rupeliensis, Hyž 14

Type figured in HYžNÝ \& DUlaI 2014: fig. 6b

Thaumastocheles rupeliensis Beurlen, 1939

paralectotypes, M.66.961

Kiscellian, Kiscell Clay Formation

Újlaki brickyard, Kiscell, Budapest

Beu39, p. 137, text-fig. 1

Ctenocheles rupeliensis, Hyž14

One of the paralectotypes figured in HYžNÝ

$\&$ Dulai 2014: fig. 4a. Type figured also in

HYŽNÝ \& KLOMPMAKER 2015: fig. 9g

\section{Miocene types}

\subsection{Miocene Bivalvia}

Cardium manyense Kókay, 1967

holotype, M.66.963

Badenian, Pusztamiske Formation?

215.10-216.90 m, borehole M-6, Mány

Kók67, p. 88 (83), pl. 7, fig. 1

Type found in the legacy of the author
Pecten fotensis subplanus Kókay in STEININGER et al., 1973

paratype, PAL 2018.26.1.

Ottnangian, Bántapuszta Formation

locality I/b, Bántapuszta, Öskü

Ste73, p. 485

Type found in the legacy of the author

\subsection{Miocene Gastropoda}

Dermomurex (Gracilimurex) nemethi Kovács, 2018

holotype, PAL 2017.55.1.

Badenian, Sámsonháza Formation

Letkés

Kov18a, p. 32, figs 2a-b

Dorsanum (Dorsanum) nodosocostatum uniseriatum Kókay in STEININGER et al., 1973

paratype, PAL 2018.27.1.

Ottnangian, Bántapuszta Formation

175.6-180.0 m, borehole V-133, Várpalota

Ste73, p. 423, pl. 6, fig. 8b

Cyllenina uniseriata, Har04

Type found in the legacy of the author

Dorsanum (Dorsanum) nodosocostatum uniseriatum Kókay in STEININGER et al., 1973

paratype, PAL 2018.28.1.

Ottnangian, Bántapuszta Formation

175.6-180.0 m, borehole V-133, Várpalota

Ste73, p. 423, pl. 6, fig. 8c

Cyllenina uniseriata, Har04

Type found in the legacy of the author

Dorsanum (Dorsanum) nodosocostatum uniseriatum Kókay in STEININGER et al., 1973

paratype, PAL 2018.29.1.

Ottnangian, Bántapuszta Formation

175.6-180.0 m, borehole V-133, Várpalota

Ste73, p. 423, pl. 6, fig. 8d

Cyllenina uniseriata, Har04

Type found in the legacy of the author

Euthria viciani Kovács, 2018

holotype, PAL 2018.1.1.

Badenian, Pusztamiske Formation

Bánd

Kov18b, p. 179, figs 2-3 
Euthria viciani Kovács, 2018

paratype, PAL 2018.2.1.

Badenian, Pusztamiske Formation

Bánd

Kov18b, p. 179, figs 8-9

Lautoconus harzhauseri Kovács in VICIÁN et al., 2017

holotype, PAL 2017.49.1.

Badenian, Sámsonháza Formation

Letkés

Vic17, p. 272, pl. 3, figs 17-18

Specimen figured in KovÁcs \& VICIÁN 2014

(figs 87-88) under inventory number INV

2013.213. as Monteiroconus mercati

Lautoconus harzhauseri Kovács in VICIÁN et al., 2017

paratype, PAL 2017.50.1.

Badenian, Sámsonháza Formation

Letkés

Vic17, p. 272, pl. 3, figs 19-20

Lautoconus harzhauseri Kovács in VICIÁN et al., 2017

paratypes, PAL 2017.51.1., PAL 2017.52.1.

Badenian, Sámsonháza Formation

Letkés

Vic17, p. 272

Leptoconus hirmetzli Kovács \& Vicián, 2014 holotype, PAL 2013.3.1.

Badenian, Sámsonháza Formation

Letkés

Kov14, p. 62, figs 34-36

Plagioconus hirmetzli, Har 16

Type figured in HARZHAUSER \& LANDAU 2016: fig. 32j

Leptoconus hirmetzli Kovács \& Vicián, 2014 paratype, PAL 2013.4.1.

Badenian, Sámsonháza Formation

Letkés

Kov14, p. 62, fig. 37

Plagioconus hirmetzli, Har 16
Leptoconus hirmetzli Kovács \& Vicián, 2014

paratypes, PAL 2013.5.1., PAL 2013.6.1., PAL

2013.7.1., PAL 2013.8.1., PAL 2013.9.1.

Badenian, Sámsonháza Formation

Letkés

Kov14, p. 62

Plagioconus hirmetzli, Har 16

Menathais viciani Kovács, 2018

holotype, PAL 2017.56.1.

Badenian, Sámsonháza Formation

Letkés

Kov18a, p. 32, figs $2 \mathrm{e}-\mathrm{f}$

Semicassis szilviae Kovács \& Vicián, 2017

holotype, PAL 2017.58.1.

Badenian, Sámsonháza Formation

Letkés

Kov17, p. 82, figs 21-22

Semicassis szilviae Kovács \& Vicián, 2017

paratypes, PAL 2017.59.1., PAL 2017.60.1.

Badenian, Sámsonháza Formation

Letkés

Kov17, p. 82

Siratus hirmetzli Kovács, 2018

holotype, PAL 2017.53.1.

Badenian, Sámsonháza Formation

Letkés

Kov18a, p. 30, figs 1a-b

Siratus hirmetzli Kovács, 2018

paratype, PAL 2017.57.1.

Badenian, Dej Formation

Lăpugiu de Sus, Romania

Kov18a, p. 30, figs 1c-d

Taurasia szobensis Csepreghy-Meznerics, 1956

holotype, M.61.4309

Badenian, Sámsonháza Formation

"large outcrop", Szob

C-M56, p. 445 (402), pl. 6, figs 1-4

Bufonaria szobensis, Rav16

Inventory number in original publication: $M$

52/253. Type figured in RAVEN 2016: pl. 1, figs

$12 \mathrm{a}-\mathrm{b}$ 
Theodoxus (Calvertia?) grateloupianus dentatus

Kókay, 1967

holotype, M.66.964

Badenian, Pusztamiske Formation

bentonite quarry, Bánd

Kók67, p. 89 (83), pl. 8, figs 1a-b

Theodoxus grateloupianus, Kók06

Type found in the legacy of the author

Valvata hidasensis Kókay, 1967

holotype, M.66.965

Badenian, Hidas Lignite Formation

Hidas

Kók67, p. 90 (84), pl. 8, figs 5a-b

Sandbergerina hidasensis, Kók06

Type found in the legacy of the author

\subsection{Miocene Brachiopoda}

Argyrotheca bitnerae Dulai in Dulai \&

STACHACZ, 2011

holotype, PAL 2011.1.1.

Badenian, Pińczów Formation

Szydłów, Poland

Dul11b, p. 285, fig. 3.1

Argyrotheca bitnerae Dulai in Dulai \&

STACHACZ, 2011

paratype, PAL 2011.2.1.

Badenian, Pińczów Formation

Szydłów, Poland

Dul11b, p. 285, fig. 3.2

Instead of the photo of the paratype, the holotype is illustrated once more by mistake

Argyrotheca bitnerae Dulai in Dulai \&

STACHACZ, 2011

paratype, PAL 2011.3.1.

Badenian, Pińczów Formation

Szydłów, Poland

Dul1 1b, p. 285, fig. 3.6

\subsection{Miocene Decapoda}

Callichirus bertalani Hyžný \& Müller, 2010

holotype, M 2009.2334.1.

Badenian, Leitha Limestone Formation

Nyirád

Hyž10, p. 41, figs 2-3

Type figured in HYžNÝ \& KLOMPMAKER

2015: fig. 10e

?Pagurus turcus Müller, 1984

holotype, 2004.164.1.

Badenian, Sámsonháza Formation

Törökmező, Nagymaros

Mül84, p. 56, pl. 13, figs 1-3

Author's inventory number: MTZ-17-1; paratypes under same inventory number (pl. 12, fig. 6, pl. 13, fig. 4). Holotype found in collection in 2013, beside specimens with intventory number M.86.153 (MTZ-21), paratypes still missing

\subsection{Miocene Echinoidea}

Scutella vindobonensis planata Kókay in Somos \& KóKAY, 1960

paratype, PAL 2018.30.1.

Badenian, Leitha Limestone Formation

new railway cut, Hird

Som60, p. 346 (341), pl. 17, fig. 3

Type found in the legacy of the author 


\section{Catalogue of vertebrate type specimens}

\section{Amphibia}

Bakonybatrachus fedori Szentesi \& Venczel, 2012

holotype, V 2010.283.1.

incomplete right ilium

Santonian, Late Cretaceous; Csehbánya

Formation

Iharkút, Bakonyjákó

Sze12, p. 328, figs 1a-d

Bakonybatrachus fedori Szentesi \& Venczel, 2012

paratype, V 2008.30.1.

fragmentary left scapula

Santonian, Late Cretaceous; Csehbánya

Formation

Iharkút, Bakonyjákó

Sze12, p. 328, figs 2e-f

Bakonybatrachus fedori Szentesi \& Venczel, 2012

paratype, V 2008.31.1.

fragmentary left angulosplenial

Santonian, Late Cretaceous; Csehbánya

Formation

Iharkút, Bakonyjákó

Sze12, p. 328, figs 2c-d

Bakonybatrachus fedori Szentesi \& Venczel, 2012

paratype, V 2009.34.1.

fragmentary right maxilla

Santonian, Late Cretaceous; Csehbánya

Formation

Iharkút, Bakonyjákó

Sze12, p. 328, figs $2 \mathrm{a}-\mathrm{b}$

Hungarobatrachus szukacsi Szentesi \& Venczel, 2010

holotype, V 2008.16.1.

incomplete right ilium

Santonian, Late Cretaceous; Csehbánya

Formation

Iharkút, Bakonyjákó

Sze10, p. 293, figs 2a-e, $4 a$
Hungarobatrachus szukacsi Szentesi \& Venczel, 2010

paratypes, V 2008.12.1., V 2008.13.1., V

2008.17.1., V 2008.18.1.

incomplete right ilia

Santonian, Late Cretaceous; Csehbánya

Formation

Iharkút, Bakonyjákó

Sze10, p. 293

Hungarobatrachus szukacsi Szentesi \& Venczel, 2010

paratype, V 2008.14.1.

incomplete left ilium

Santonian, Late Cretaceous; Csehbánya

Formation

Iharkút, Bakonyjákó

Sze10, p. 293, figs 3d-e

Hungarobatrachus szukacsi Szentesi \& Venczel, 2010

paratype, V 2008.15.1.

incomplete right ilium

Santonian, Late Cretaceous; Csehbánya

Formation

Iharkút, Bakonyjákó

Sze10, p. 293, figs 3a-c

Hungarobatrachus szukacsi Szentesi \& Venczel, 2010

paratypes, V 2008.19.1., V 2008.21.1.

fragmentary tibio-fibulae

Santonian, Late Cretaceous; Csehbánya

Formation

Iharkút, Bakonyjákó

Sze10, p. 293

Hungarobatrachus szukacsi Szentesi \& Venczel, 2010

paratype, V 2008.32.1.

right tibio-fibula

Santonian, Late Cretaceous; Csehbánya

Formation

Iharkút, Bakonyjákó

Sze10, p. 293, figs $3 f-g$ 
Hungarobatrachus szukacsi Szentesi \& Venczel, 2010

paratype, PAL 2018.31.1.

fragmentary tibio-fibula

Santonian, Late Cretaceous; Csehbánya

Formation

Iharkút, Bakonyjákó

Sze10, p. 293

Invalid inventory number given in original

publication as V2008.33.1

Hungarobatrachus szukacsi Szentesi \& Venczel, 2010

paratype, PAL 2018.32.1.

fragmentary tibio-fibula

Santonian, Late Cretaceous; Csehbánya

Formation

Iharkút, Bakonyjákó

Sze10, p. 293

Invalid inventory number given in original publication as V2008.34.1

\section{Reptilia}

Ajkaceratops kozmai Ösi, Butler \&

Weishampel, 2010

holotype, V 2009.192.1.

fused premaxillae and rostral bones with fragments of the maxillae

Santonian, Late Cretaceous; Csehbánya

Formation

Iharkút, Bakonyjákó

Ösi10b, p. 466, figs 1a-b

Ajkaceratops kozmai Ösi, Butler \&

Weishampel, 2010

paratype, V 2009.193.1.

predentary bone

Santonian, Late Cretaceous; Csehbánya

Formation

Iharkút, Bakonyjákó

Ösi10b, p. 466, figs 1c-e
Ajkaceratops kozmai Ösi, Butler \&

Weishampel, 2010

paratypes, V 2009.194.1., V 2009.195.1., V

2009.196.1.

predentary bones

Santonian, Late Cretaceous; Csehbánya

Formation

Iharkút, Bakonyjákó

Ösi10b, p. 466

Chromatogenys tiliquoides Makádi \& Nydam, 2015

holotype, V 2010.129.1.

partial right mandible

Santonian, Late Cretaceous; Csehbánya

Formation

Iharkút, Bakonyjákó

Mak15, p. 928, figs 2a-c, 3a-c

Distortodon rhomboideus Makádi, 2013

holotype, PAL 2012.31.1.

partial right maxilla

Santonian, Late Cretaceous; Csehbánya

Formation

Iharkút, Bakonyjákó

Mak13a, p. 168, figs 2a-e

Distortodon rhomboideus Makádi, 2013

paratype, PAL 2012.32.1.

right dentary

Santonian, Late Cretaceous; Csehbánya

Formation

Iharkút, Bakonyjákó

Mak13a, p. 168, figs 3a-e

Distortodon rhomboideus Makádi, 2013

paratype, PAL 2012.33.1.

partial left dentary

Santonian, Late Cretaceous; Csehbánya

Formation

Iharkút, Bakonyjákó

Mak13a, p. 168, figs 4a-e

Foxemys trabanti Rabi, Tong \& Botfalvai, 2012

holotype, V 2010.86.1.

incomplete skull

Santonian, Late Cretaceous; Csehbánya

Formation

Iharkút, Bakonyjákó

Rab12, p. 663, figs 2a-b, 3a-f 
Foxemys trabanti Rabi, Tong \& Botfalvai, 2012 paratype, V 2010.87.1.

partial skull

Santonian, Late Cretaceous; Csehbánya

Formation

Iharkút, Bakonyjákó

Rab12, p. 663, figs 4a-d

Foxemys trabanti Rabi, Tong \& Botfalvai, 2012 paratype, V 2010.88.1.

left otic chamber

Santonian, Late Cretaceous; Csehbánya

Formation

Iharkút, Bakonyjákó

Rab12, p. 663, figs 5a-f

Foxemys trabanti Rabi, Tong \& Botfalvai, 2012 paratype, V 2010.89.1.

fragmentary left lower jaw

Santonian, Late Cretaceous; Csehbánya

Formation

Iharkút, Bakonyjákó

Rab12, p. 663, figs 2c-d

Foxemys trabanti Rabi, Tong \& Botfalvai, 2012 paratype, V 2010.215.1.

skull

Santonian, Late Cretaceous; Csehbánya

Formation

Iharkút, Bakonyjákó

Rab12, p. 663

Foxemys trabanti Rabi, Tong \& Botfalvai, 2012 paratype, V 2010.216.1.

partial skull

Santonian, Late Cretaceous; Csehbánya

Formation

Iharkút, Bakonyjákó

Rab12, p. 663

Foxemys trabanti Rabi, Tong \& Botfalvai, 2012 paratype, V 2010.217.1.

partial skull

Santonian, Late Cretaceous; Csehbánya

Formation

Iharkút, Bakonyjákó

Rab12, p. 663

Specimen given in original publication as uncatalogued
Foxemys trabanti Rabi, Tong \& Botfalvai, 2012 paratype, V 2010.219.1.

right and left lower jaw

Santonian, Late Cretaceous; Csehbánya

Formation

Iharkút, Bakonyjákó

Rab12, p. 663

Foxemys trabanti Rabi, Tong \& Botfalvai, 2012 paratype, V 2010.220.1.

left lower jaw

Santonian, Late Cretaceous; Csehbánya

Formation

Iharkút, Bakonyjákó

Rab12, p. 663

Magyarosuchus fitosi Ösi, Young, Galácz \&

Rabi, 2018

holotype, V.97.1

posterior tooth

Toarcian, Early Jurassic; Kisgerecse Marl

Formation

Nagy-Pisznice, Lábatlan

Ösi18, p. 6, figs 4c-f

Magyarosuchus fitosi Ösi, Young, Galácz \& Rabi, 2018

holotype, V.97.2A

middle third of left dentary

Toarcian, Early Jurassic; Kisgerecse Marl

Formation

Nagy-Pisznice, Lábatlan

Ösi18, p. 6, figs 3a-b

Magyarosuchus fitosi Ösi, Young, Galácz \&

Rabi, 2018

holotype, V.97.2B

posterior third of left dentary

Toarcian, Early Jurassic; Kisgerecse Marl

Formation

Nagy-Pisznice, Lábatlan

Ösi18, p. 6, figs 3c-e 
Magyarosuchus fitosi Ösi, Young, Galácz \& Rabi, 2018

holotype, V.97.2C

mandible fragment

Toarcian, Early Jurassic; Kisgerecse Marl

Formation

Nagy-Pisznice, Lábatlan

Ösi18, p. 6, figs 3j-1

Magyarosuchus fitosi Ösi, Young, Galácz \& Rabi, 2018

holotype, V.97.4, V.97.24, V.97.53, V.97.56

teeth, fragmentary osteoderms

Toarcian, Early Jurassic; Kisgerecse Marl

Formation

Nagy-Pisznice, Lábatlan

Ösi18, p. 6

Magyarosuchus fitosi Ösi, Young, Galácz \& Rabi, 2018

holotype, V.97.5, V.97.55

teeth

Toarcian, Early Jurassic; Kisgerecse Marl

Formation

Nagy-Pisznice, Lábatlan

Ösi18, p. 6

Magyarosuchus fitosi Ösi, Young, Galácz \& Rabi, 2018

holotype, V.97.7

right coracoid

Toarcian, Early Jurassic; Kisgerecse Marl

Formation

Nagy-Pisznice, Lábatlan

Ösi18, p. 6, figs 6g-h

Magyarosuchus fitosi Ösi, Young, Galácz \&

Rabi, 2018

holotype, V.97.8

fragmentary dorsal rib

Toarcian, Early Jurassic; Kisgerecse Marl

Formation

Nagy-Pisznice, Lábatlan

Ösi18, p. 6, figs 6a-b
Magyarosuchus fitosi Ösi, Young, Galácz \&

Rabi, 2018

holotype, V.97.9

left tibia

Toarcian, Early Jurassic; Kisgerecse Marl

Formation

Nagy-Pisznice, Lábatlan

Ösi18, p. 6, figs 7j-o

Magyarosuchus fitosi Ösi, Young, Galácz \& Rabi, 2018

holotype, V.97.10

metatarsal III

Toarcian, Early Jurassic; Kisgerecse Marl

Formation

Nagy-Pisznice, Lábatlan

Ösi18, p. 6, figs 8g-j

Magyarosuchus fitosi Ösi, Young, Galácz \& Rabi, 2018

holotype, V.97.11, V.97.45

metatarsals

Toarcian, Early Jurassic; Kisgerecse Marl

Formation

Nagy-Pisznice, Lábatlan

Ösi18, p. 6

Magyarosuchus fitosi Ösi, Young, Galácz \& Rabi, 2018

holotype, V.97.12

left astragalus

Toarcian, Early Jurassic; Kisgerecse Marl

Formation

Nagy-Pisznice, Lábatlan

Ösi18, p. 6, figs 8a-f

Magyarosuchus fitosi Ösi, Young, Galácz \&

Rabi, 2018

holotype, V.97.13

left femur

Toarcian, Early Jurassic; Kisgerecse Marl

Formation

Nagy-Pisznice, Lábatlan

Ösi18, p. 6, figs 7d-i 
Magyarosuchus fitosi Ösi, Young, Galácz \& Rabi, 2018

holotype, V.97.14, V.97.16, V.97.17, V.97.46, V.97.47, V.97.48, V.97.51, V.97.52, V.97.54, V.97.64, V.97.67, V.97.68

dorsal rib fragments

Toarcian, Early Jurassic; Kisgerecse Marl

Formation

Nagy-Pisznice, Lábatlan

Ösi18, p. 6

Magyarosuchus fitosi Ösi, Young, Galácz \& Rabi, 2018

holotype, V.97.15

proximal end of fibula, dorsal rib fragments

Toarcian, Early Jurassic; Kisgerecse Marl

Formation

Nagy-Pisznice, Lábatlan

Ösi18, p. 6, figs 7p-r

Magyarosuchus fitosi Ösi, Young, Galácz \& Rabi, 2018

holotype, V.97.18, V.97.65

ventral osteoderms

Toarcian, Early Jurassic; Kisgerecse Marl

Formation

Nagy-Pisznice, Lábatlan

Ösi18, p. 6

Magyarosuchus fitosi Ösi, Young, Galácz \&

Rabi, 2018

holotype, V.97.19

distal caudal vertebra

Toarcian, Early Jurassic; Kisgerecse Marl

Formation

Nagy-Pisznice, Lábatlan

Ösi18, p. 6, figs 5k-p, $12 \mathrm{i}$

Magyarosuchus fitosi Ösi, Young, Galácz \&

Rabi, 2018

holotype, V.97.21, V.97.22

distal caudal vertebrae

Toarcian, Early Jurassic; Kisgerecse Marl

Formation

Nagy-Pisznice, Lábatlan

Ösi18, p. 6
Magyarosuchus fitosi Ösi, Young, Galácz \&

Rabi, 2018

holotype, V.97.26

dorsal vertebrae

Toarcian, Early Jurassic; Kisgerecse Marl

Formation

Nagy-Pisznice, Lábatlan

Ösi18, p. 6, figs 5a-c

Magyarosuchus fitosi Ösi, Young, Galácz \& Rabi, 2018

holotype, V.97.27

caudal vertebrae, sacral rib

Toarcian, Early Jurassic; Kisgerecse Marl

Formation

Nagy-Pisznice, Lábatlan

Ösi18, p. 6

Magyarosuchus fitosi Ösi, Young, Galácz \& Rabi, 2018

holotype, V.97.28

mid-caudal vertebrae

Toarcian, Early Jurassic; Kisgerecse Marl

Formation

Nagy-Pisznice, Lábatlan

Ösi18, p. 6, figs 5f-h

Magyarosuchus fitosi Ösi, Young, Galácz \& Rabi, 2018

holotype, V.97.29

tooth, proximal caudal vertebra

Toarcian, Early Jurassic; Kisgerecse Marl

Formation

Nagy-Pisznice, Lábatlan

Ösi18, p. 6

Magyarosuchus fitosi Ösi, Young, Galácz \&

Rabi, 2018

holotype, V.97.30

sacrum with the last dorsal and the first caudal vertebra, two sacral vertebrae

Toarcian, Early Jurassic; Kisgerecse Marl

Formation

Nagy-Pisznice, Lábatlan

Ösi18, p. 6, figs 5d-e 
Magyarosuchus fitosi Ösi, Young, Galácz \& Rabi, 2018

holotype, V.97.31

distal caudal vertebrae

Toarcian, Early Jurassic; Kisgerecse Marl

Formation

Nagy-Pisznice, Lábatlan

Ösi18, p. 6, figs $5 \mathrm{i}-\mathrm{j}$

Magyarosuchus fitosi Ösi, Young, Galácz \&

Rabi, 2018

holotype, V.97.33

right femur

Toarcian, Early Jurassic; Kisgerecse Marl

Formation

Nagy-Pisznice, Lábatlan

Ösi18, p. 6

Magyarosuchus fitosi Ösi, Young, Galácz \& Rabi, 2018

holotype, V.97.34

left ilium

Toarcian, Early Jurassic; Kisgerecse Marl

Formation

Nagy-Pisznice, Lábatlan

Ösi18, p. 6, figs 6i-1

Magyarosuchus fitosi Ösi, Young, Galácz \&

Rabi, 2018

holotype, V.97.35

left pubis

Toarcian, Early Jurassic; Kisgerecse Marl

Formation

Nagy-Pisznice, Lábatlan

Ösi18, p. 6, figs 6n-o

Magyarosuchus fitosi Ösi, Young, Galácz \&

Rabi, 2018

holotype, V.97.36

left ischium

Toarcian, Early Jurassic; Kisgerecse Marl

Formation

Nagy-Pisznice, Lábatlan

Ösi18, p. 6, fig. 6p
Magyarosuchus fitosi Ösi, Young, Galácz \&

Rabi, 2018

holotype, V.97.37

sacral rib with crest

Toarcian, Early Jurassic; Kisgerecse Marl

Formation

Nagy-Pisznice, Lábatlan

Ösi18, p. 6, figs 6c-d

Magyarosuchus fitosi Ösi, Young, Galácz \&

Rabi, 2018

holotype, V.97.38

a short limb bone associated with a dorsal rib and a third bone fragment, ventral osteoderms

Toarcian, Early Jurassic; Kisgerecse Marl

Formation

Nagy-Pisznice, Lábatlan

Ösi18, p. 6, figs 7c, 9g-h, 9i

Magyarosuchus fitosi Ösi, Young, Galácz \&

Rabi, 2018

holotype, V.97.39

sacral rib

Toarcian, Early Jurassic; Kisgerecse Marl

Formation

Nagy-Pisznice, Lábatlan

Ösi18, p. 6, figs 6e-f

Magyarosuchus fitosi Ösi, Young, Galácz \&

Rabi, 2018

holotype, V.97.40

left angular-surangular

Toarcian, Early Jurassic; Kisgerecse Marl

Formation

Nagy-Pisznice, Lábatlan

Ösi18, p. 6, figs 3f-i

Magyarosuchus fitosi Ösi, Young, Galácz \&

Rabi, 2018

holotype, V.97.41

fibula?

Toarcian, Early Jurassic; Kisgerecse Marl

Formation

Nagy-Pisznice, Lábatlan

Ösi18, p. 6 
Magyarosuchus fitosi Ősi, Young, Galácz \& Rabi, 2018

holotype, V.97.42

proximal end of radius

Toarcian, Early Jurassic; Kisgerecse Marl

Formation

Nagy-Pisznice, Lábatlan

Ösi18, p. 6, figs 7a-b

Magyarosuchus fitosi Ösi, Young, Galácz \& Rabi, 2018

holotype, V.97.43

distal end of fibula

Toarcian, Early Jurassic; Kisgerecse Marl

Formation

Nagy-Pisznice, Lábatlan

Ösi18, p. 6, figs 7s-t

Magyarosuchus fitosi Ősi, Young, Galácz \& Rabi, 2018

holotype, V.97.44

right ilium

Toarcian, Early Jurassic; Kisgerecse Marl

Formation

Nagy-Pisznice, Lábatlan

Ösi18, p. 6, fig. 6m

Magyarosuchus fitosi Ősi, Young, Galácz \& Rabi, 2018

holotype, V.97.49

distal half of right pubis

Toarcian, Early Jurassic; Kisgerecse Marl

Formation

Nagy-Pisznice, Lábatlan

Ösi18, p. 6

Magyarosuchus fitosi Ösi, Young, Galácz \&

Rabi, 2018

holotype, V.97.50, V.97.58

other fragmentary elements

Toarcian, Early Jurassic; Kisgerecse Marl

Formation

Nagy-Pisznice, Lábatlan

Ösi18, p. 6
Magyarosuchus fitosi Ösi, Young, Galácz \&

Rabi, 2018

holotype, V.97.57

anterior tooth, anterior or middle tooth, mid-

dle or posterior tooth

Toarcian, Early Jurassic; Kisgerecse Marl

Formation

Nagy-Pisznice, Lábatlan

Ösi18, p. 6, figs 4a, 4b, 4g-i

Magyarosuchus fitosi Ösi, Young, Galácz \&

Rabi, 2018

holotype, V.97.59

dorsal osteoderm

Toarcian, Early Jurassic; Kisgerecse Marl

Formation

Nagy-Pisznice, Lábatlan

Ösi18, p. 6, figs 9a-c

Magyarosuchus fitosi Ösi, Young, Galácz \&

Rabi, 2018

holotype, V.97.60

dorsal osteoderms, other fragmentary elements

Toarcian, Early Jurassic; Kisgerecse Marl

Formation

Nagy-Pisznice, Lábatlan

Ösi18, p. 6, figs 9d, 9e-f

Magyarosuchus fitosi Ösi, Young, Galácz \&

Rabi, 2018

holotype, V.97.61

phalanx

Toarcian, Early Jurassic; Kisgerecse Marl

Formation

Nagy-Pisznice, Lábatlan

Ösi18, p. 6, figs 8k-1

Magyarosuchus fitosi Ösi, Young, Galácz \&

Rabi, 2018

holotype, V.97.66

unidentified limb bone element

Toarcian, Early Jurassic; Kisgerecse Marl

Formation

Nagy-Pisznice, Lábatlan

Ösi18, p. 6, fig. $8 \mathrm{~m}$

No inventory number given in original publi-

cation 
Magyarosuchus fitosi Ösi, Young, Galácz \& Rabi, 2018

holotype, V.97.69

right tibia

Toarcian, Early Jurassic; Kisgerecse Marl

Formation

Nagy-Pisznice, Lábatlan

Ösi18, p. 6

Mochlodon vorosi Ösi, Prondvai, Butler \&

Weishampel, 2012

holotype, V 2010.105.1.

left complete dentary with four broken teeth

Santonian, Late Cretaceous; Csehbánya

Formation

Iharkút, Bakonyjákó

Ösi12, p. 6, figs 2f-h, 3g-h

Mochlodon vorosi Ösi, Prondvai, Butler \&

Weishampel, 2012

paratype, V.2000.1

dentary tooth

Santonian, Late Cretaceous; Csehbánya

Formation

Iharkút, Bakonyjákó

Ösi12, p. 6

Mochlodon vorosi Ösi, Prondvai, Butler \&

Weishampel, 2012

paratypes, V.2000.33, V.2001.64, V.2001.161

maxillary teeth

Santonian, Late Cretaceous; Csehbánya

Formation

Iharkút, Bakonyjákó

Ösi12, p. 6

Mochlodon vorosi Ösi, Prondvai, Butler \&

Weishampel, 2012

paratype, V.2001.53

incomplete left coracoid

Santonian, Late Cretaceous; Csehbánya

Formation

Iharkút, Bakonyjákó

Ösi12, p. 6, figs 6a-c
Mochlodon vorosi Ösi, Prondvai, Butler \&

Weishampel, 2012

paratype, V.2001.101

fragmentary left tibia

Santonian, Late Cretaceous; Csehbánya

Formation

Iharkút, Bakonyjákó

Ösi12, p. 6, figs 9e-f

Mochlodon vorosi Ösi, Prondvai, Butler \&

Weishampel, 2012

paratype, V.2001.225

almost complete left femur

Santonian, Late Cretaceous; Csehbánya

Formation

Iharkút, Bakonyjákó

Ösi12, p. 6, figs 7a-e, 9c-d

Mochlodon vorosi Ösi, Prondvai, Butler \& Weishampel, 2012

paratypes, V 2010.106.1., V 2010.108.1., V

2010.112.1., PAL 2012.16.1.

fragmentary dentaries

Santonian, Late Cretaceous; Csehbánya

Formation

Iharkút, Bakonyjákó

Ösi12, p. 6

Mochlodon vorosi Ösi, Prondvai, Butler \&

Weishampel, 2012

paratype, V 2010.107.1.

fragmentary right dentary

Santonian, Late Cretaceous; Csehbánya

Formation

Iharkút, Bakonyjákó

Ösi12, p. 6

Mochlodon vorosi Ösi, Prondvai, Butler \&

Weishampel, 2012

paratype, V 2010.109.1.

fragmentary right dentary

Santonian, Late Cretaceous; Csehbánya

Formation

Iharkút, Bakonyjákó

Ösi12, p. 6, figs 3i-j 
Mochlodon vorosi Ösi, Prondvai, Butler \&

Weishampel, 2012

paratype, V 2010.110.1.

right quadrate

Santonian, Late Cretaceous; Csehbánya

Formation

Iharkút, Bakonyjákó

Ösi12, p. 6

Mochlodon vorosi Ösi, Prondvai, Butler \&

Weishampel, 2012

paratype, V 2010.111.1.

right quadrate

Santonian, Late Cretaceous; Csehbánya

Formation

Iharkút, Bakonyjákó

Ösi12, p. 6, figs 2a-e

Mochlodon vorosi Ösi, Prondvai, Butler \&

Weishampel, 2012

paratype, V 2010.118.1.

isolated dorsal vertebra

Santonian, Late Cretaceous; Csehbánya

Formation

Iharkút, Bakonyjákó

Ösi12, p. 6, figs 5c-f

Mochlodon vorosi Ösi, Prondvai, Butler \&

Weishampel, 2012

paratype, V 2010.121.1.

almost complete but compressed sacrum

Santonian, Late Cretaceous; Csehbánya

Formation

Iharkút, Bakonyjákó

Ösi12, p. 6, figs 5g-j

Inventory number given erroneously in figure

caption as 2010.118.1.

Mochlodon vorosi Ösi, Prondvai, Butler \&

Weishampel, 2012

paratypes, V 2010.122.1., V 2010.123.1.

incomplete left coracoids

Santonian, Late Cretaceous; Csehbánya

Formation

Iharkút, Bakonyjákó

Ösi12, p. 6
Mochlodon vorosi Ösi, Prondvai, Butler \&

Weishampel, 2012

paratype, V 2010.126.1.

almost complete left femur

Santonian, Late Cretaceous; Csehbánya

Formation

Iharkút, Bakonyjákó

Ösi12, p. 6, figs 10a-b

Mochlodon vorosi Ösi, Prondvai, Butler \& Weishampel, 2012

paratype, V 2010.127.1.

complete right tibia

Santonian, Late Cretaceous; Csehbánya

Formation

Iharkút, Bakonyjákó

Ösi12, p. 6, figs $7 f-i$

Mochlodon vorosi Ösi, Prondvai, Butler \&

Weishampel, 2012

paratype, V 2010.128.1.

complete right humerus

Santonian, Late Cretaceous; Csehbánya

Formation

Iharkút, Bakonyjákó

Ösi12, p. 6, figs 6g-k

Mochlodon vorosi Ösi, Prondvai, Butler \&

Weishampel, 2012

paratype, PAL 2012.14.1.

left postorbital

Santonian, Late Cretaceous; Csehbánya

Formation

Iharkút, Bakonyjákó

Ösi12, p. 6, figs $2 \mathrm{i}-\mathrm{k}$

Mochlodon vorosi Ösi, Prondvai, Butler \&

Weishampel, 2012

paratype, PAL 2012.15.1.

left dentary

Santonian, Late Cretaceous; Csehbánya

Formation

Iharkút, Bakonyjákó

Ösi12, p. 6 
Mochlodon vorosi Ösi, Prondvai, Butler \&

Weishampel, 2012

paratypes, PAL 2012.17.1.

five maxillary teeth

Santonian, Late Cretaceous; Csehbánya

Formation

Iharkút, Bakonyjákó

Ösi12, p. 6, figs 4d-e, 4f-g

Types figured in VIRÁG \& Ösı 2017: figs 2d-e (\#36A), figs $2 \mathrm{k}-\mathrm{m}(\# 36 \mathrm{E})$

Mochlodon vorosi Ösi, Prondvai, Butler \& Weishampel, 2012

paratypes, PAL 2012.18.1.

five dentary teeth

Santonian, Late Cretaceous; Csehbánya

Formation

Iharkút, Bakonyjákó

Ösi12, p. 6, figs 4a-c

Types figured in VirÁg \& Ösi 2017: figs

$3 \mathrm{~m}-\mathrm{n}, 5 \mathrm{e}-\mathrm{f}$ (\#35A), figs 3o-r (\#35C), figs

6d-e, 7d-e (\#35D). Specimen \#35C referred to

Ajkaceratops kozmai in VIRÁG \& Ösı 2017

Mochlodon vorosi Ösi, Prondvai, Butler \&

Weishampel, 2012

paratype, PAL 2012.19.1.

isolated cervical vertebra

Santonian, Late Cretaceous; Csehbánya

Formation

Iharkút, Bakonyjákó

Ösi12, p. 6, figs 5a-b

Mochlodon vorosi Ösi, Prondvai, Butler \&

Weishampel, 2012

paratype, PAL 2012.20.1.

isolated caudal vertebra

Santonian, Late Cretaceous; Csehbánya

Formation

Iharkút, Bakonyjákó

Ösi12, p. 6, figs 5k-m

Mochlodon vorosi Ösi, Prondvai, Butler \&

Weishampel, 2012

paratype, PAL 2012.21.1.

isolated caudal vertebra

Santonian, Late Cretaceous; Csehbánya

Formation

Iharkút, Bakonyjákó

Ösi12, p. 6, figs 5n-p
Mochlodon vorosi Ösi, Prondvai, Butler \&

Weishampel, 2012

paratype, PAL 2012.22.1.

incomplete left scapula

Santonian, Late Cretaceous; Csehbánya

Formation

Iharkút, Bakonyjákó

Ösi12, p. 6, figs 6d-f

Mochlodon vorosi Ösi, Prondvai, Butler \&

Weishampel, 2012

paratype, PAL 2012.23.1.

fragmentary left humerus

Santonian, Late Cretaceous; Csehbánya

Formation

Iharkút, Bakonyjákó

Ösi12, p. 6, figs 9a-b

Mochlodon vorosi Ösi, Prondvai, Butler \&

Weishampel, 2012

paratype, PAL 2012.24.1.

complete right ulna

Santonian, Late Cretaceous; Csehbánya

Formation

Iharkút, Bakonyjákó

Ösi12, p. 6, figs 61-o

Mochlodon vorosi Ösi, Prondvai, Butler \&

Weishampel, 2012

paratype, PAL 2012.25.1.

fragmentary right femur

Santonian, Late Cretaceous; Csehbánya

Formation

Iharkút, Bakonyjákó

Ösi12, p. 6, figs 10c-d

Mochlodon vorosi Ösi, Prondvai, Butler \&

Weishampel, 2012

paratype, PAL 2012.26.1.

fragmentary left tibia

Santonian, Late Cretaceous; Csehbánya

Formation

Iharkút, Bakonyjákó

Ösi12, p. 6, figs 10e-f 
Mochlodon vorosi Ösi, Prondvai, Butler \&

Weishampel, 2012

paratypes, PAL 2012.27.1., PAL 2012.28.1. phalanges

Santonian, Late Cretaceous; Csehbánya

Formation

Iharkút, Bakonyjákó

Ösi12, p. 6

Mochlodon vorosi Ösi, Prondvai, Butler \&

Weishampel, 2012

paratype, PAL 2014.114.1.

maxillary tooth

Santonian, Late Cretaceous; Csehbánya

Formation

Iharkút, Bakonyjákó

Ösi12, p. 6

Invalid inventory number given in original publication as V 2003.15

Mochlodon vorosi Ösi, Prondvai, Butler \&

Weishampel, 2012

paratype, PAL 2014.118.1.

dentary tooth

Santonian, Late Cretaceous; Csehbánya

Formation

Iharkút, Bakonyjákó

Ösi12, p. 6

Invalid inventory number given in original publication as V 2003.10.

Mochlodon vorosi Ösi, Prondvai, Butler \&

Weishampel, 2012

paratype, PAL 2014.119.1.

maxillary tooth

Santonian, Late Cretaceous; Csehbánya

Formation

Iharkút, Bakonyjákó

Ösi12, p. 6

Invalid inventory number given in original publication as V 2003.14
Mochlodon vorosi Ösi, Prondvai, Butler \&

Weishampel, 2012

paratype, PAL 2014.120.1.

maxillary tooth

Santonian, Late Cretaceous; Csehbánya

Formation

Iharkút, Bakonyjákó

Ösi12, p. 6

Invalid inventory number given in original publication as V.2003.16

Mochlodon vorosi Ösi, Prondvai, Butler \&

Weishampel, 2012

paratype, PAL 2018.33.1.

maxillary tooth

Santonian, Late Cretaceous; Csehbánya

Formation

Iharkút, Bakonyjákó

Ösi12, p. 6

Invalid inventory number given in original publication as V 2000.32.

Pannoniasaurus inexpectatus Makádi, Caldwell \& Ösi, 2012

holotype, PAL 2011.43.1.

isolated right quadrate

Santonian, Late Cretaceous; Csehbánya

Formation

Iharkút, Bakonyjákó

Mak12, p. 6, figs 3a-f, $4 \mathrm{f}$

Pannoniasaurus inexpectatus Makádi, Caldwell \& Ösi, 2012

paratype, V.2001.115

left quadrate

Santonian, Late Cretaceous; Csehbánya

Formation

Iharkút, Bakonyjákó

Mak12, p. 6

Pannoniasaurus inexpectatus Makádi, Caldwell \& Ösi, 2012

paratype, PAL 2014.8.1.

fragmentary left quadrate

Santonian, Late Cretaceous; Csehbánya

Formation

Iharkút, Bakonyjákó

Mak12, p. 6

Invalid inventory number given in original

publication as 2007.31.1. 
Pelsochamops infrequens Makádi, 2013

holotype, 2006.106.1.

partial left mandible

Santonian, Late Cretaceous; Csehbánya

Formation

Iharkút, Bakonyjákó

Mak13b, p. 267, figs 2a-b, 3

Pelsochamops infrequens Makádi, 2013

paratype, PAL 2013.24.1.

right dentary fragment

Santonian, Late Cretaceous; Csehbánya

Formation

Iharkút, Bakonyjákó

Mak13b, p. 267, figs 4a-c

Pneumatoraptor fodori Ösi, Apesteguía \&

Kowalewski, 2010

holotype, V 2008.38.1.

nearly complete left scapulocoracoid

Santonian, Late Cretaceous; Csehbánya

Formation

Iharkút, Bakonyjákó

Ösi10a, p. 308, figs 4a-d

\section{Aves}

Bauxitornis mindszentyae Dyke \& Ösi, 2010 holotype, V 2009.38.1.

almost complete right tarsometatarsus

Santonian, Late Cretaceous; Csehbánya

Formation

Iharkút, Bakonyjákó

Dyk10, p. 436, figs 2a-f, $5 \mathrm{~m}$

Cuculus pannonicus Kessler, 2010

holotype, V 2009.51.1.

distal fragment of right humerus

Late Pliocene

quarry, locality 15 , Beremend

Kes10, p. 53 (56), figs 1a-b

Heliadornis minor Kessler, 2009

holotype, V 2008.44.1.

distal epiphysis of right humerus

Late Pliocene

Hajnáčka (Ajnácskő), Slovakia

Kes09, p. 67 (71), figs 2a-b

\section{Mammalia}

Allocricetus éhiki Schaub, 1930

syntypes, V.61.1522

two mandibles

Middle Pliocene

quarry, Beremend

ScS30, p. 34

Types found in collection in 2013

Archidiskodon meridionalis ürömensis Vörös, 1979

holotype, V.72.116

left $\mathrm{M}^{3}$

Middle Pleistocene

Üröm-hegy, Budapest

VöI79, p. 5, pl. 1

Mammuthus trogontherii, Vir09

Type figured in VIRÁG 2009: pl. 1, figs 1a-c

Archidiskodon meridionalis ürömensis Vörös, 1979

paratype, V.59.913

aboral fragment of left $\mathrm{M}^{2}$

Early Pleistocene

fissure fill in quarry, Gombasek (Gombaszög),

Slovakia

VöI79, p. 5

Mammuthus sp., Vir09

Type figured in Virág 2009: pl. 1, figs 2a-b

Hystrix vinogradovi atavus Jánossy, 1972

paratype, V.73.69

left mandible with $\mathrm{D}_{4}-\mathrm{M}_{3}$

Early Pleistocene

Osztramos, locality 8, Tornaszentandrás

Ján72, p. 173, pl. 1, figs 3-4

Type found in collection in 2013 
Mastodon (Bunolophodon) grandincisivum

Schlesinger, 1917

syntype, V.79.34

one upper and two lower tusks, left and right

$\mathrm{M}^{3}$, mandible fragments with left and right

$M_{3}$, postcranial skeletal parts, right scapula

fragment, humerus fragments, ulna and radius

fragments, left and right magnum, right trap-

ezoideum, right intermedium, right unciforme, metapodium fragment, phalanx fragment, left and right pelvis fragments, femur fragments, right tibia, patella, calcaneus, rib fragments, vertebrae

Pannonian, Miocene

Pestszentlörinc, Budapest

ScG17, p. 119

Konobelodon atticus, KRT14

Type figured and described in detail in

SCHLESINGER 1922: p. 7, pl. 1, figs 1-2, pl.

2 , figs 1-3, pl. 3, figs 1-7, pl. 4, figs 1-3, pl.

5 , figs $1-5$, pl. 6 , fig. 1 , original species name emended as grandincisivus. Left mandible fragment with $\mathrm{M}_{3}$ figured in GASPARIK 2007: pl.

2, fig. 6. Cross-section of lower tusk figured in KONIDARIs et al.2014: fig. 6h

"Pliomys" progressus Kretzoi, 1938

holotype, V.59.1054A

mandible

Early Pleistocene

fissure fill in quarry, Gombasek (Gombaszög),

Slovakia

Kre38, p. 96, text-fig. 2k

Inventory number in original publication: Fa.

17. Type found in collection in 2013

“Pliomys" progressus Kretzoi, 1938

paratype, V.59.1054B

fragmentary mandible

Early Pleistocene

fissure fill in quarry, Gombasek (Gombaszög),

Slovakia

Kre38, p. 96

Type found in collection in 2013. Another

paratype (mandible) still missing
Trilophodon angustidens praetypica Tasnádi

Kubacska, 1939

syntype, 2007.96.1.

maxilla fragment with left and right $\mathrm{I}^{2}$

Ottnangian, Miocene; Salgótarján Formation

coal mine, Zagyvapálfalva, Salgótarján

Tas39, p. 154, text-fig. 1

Gomphotherium praetypicum, Gas09

Type figured in GASPARIK \& MAR KOV 2009: pl. 1 , fig. 9

Trilophodon angustidens praetypica Tasnádi

Kubacska, 1939

syntype, 2007.96.2.

left mandible with $\mathrm{I}_{2}, \mathrm{P}_{4}-\mathrm{M}_{2}$

Ottnangian, Miocene; Salgótarján Formation

coal mine, Zagyvapálfalva, Salgótarján

Tas39, p. 154, pl. 4

Gomphotherium praetypicum, Gas09

Type figured in GASPARIK \& MARKOV 2009: text-fig. 3, pl. 1, figs 1-4, 8

Trilophodon angustidens praetypica Tasnádi

Kubacska, 1939

syntype, 2007.96.3.

right mandible with $\mathrm{I}_{2}, \mathrm{P}_{4}-\mathrm{M}_{2}$

Ottnangian, Miocene; Salgótarján Formation

coal mine, Zagyvapálfalva, Salgótarján

Tas39, p. 154, pl. 4

Gomphotherium praetypicum, Gas09

Type figured in GASPARIK \& MAR KOV 2009: text-fig. 3, pl. 1, figs 1-7

Ursus etruscus gombaszögensis Kretzoi, 1938

holotype, V.59.930

right $\mathrm{M}_{2}$

Early Pleistocene

fissure fill in quarry, Gombasek (Gombaszög),

Slovakia

Kre38, p. 138

Ursus deningeri gombaszogensis, Wag 14

Inventory number in original publication: $\mathrm{Fa}$.

21. Type figured in WAgNER \& GASPARIK

2014: figs 2.1a-b 
Ursus etruscus gombaszögensis Kretzoi, 1938

paratype, V.59.932

right $\mathrm{M}_{2}$

Early Pleistocene

fissure fill in quarry, Gombasek (Gombaszög),

Slovakia

Kre38, p. 138

Ursus deningeri gombaszogensis, Wag 14

Inventory number in original publication: Fa. 85

Ursus etruscus gombaszögensis Kretzoi, 1938

paratype, V.59.1048

right $\mathrm{M}_{2}$

Early Pleistocene

fissure fill in quarry, Gombasek (Gombaszög),

Slovakia

Kre38, p. 138, pl. 3, fig. 15

Ursus deningeri gombaszogensis, Wag14

Inventory number in original publication: $\mathrm{V}$.

883. Type figured in WAGNER \& GASPARIK

2014: figs 2.3a-b 


\section{INDEX OF SPECIES AND SUBSPECIES NAMES}

\begin{tabular}{|c|c|}
\hline agostyanense & Simoceras agostyani Főzy \& Scherzinger 25 \\
\hline alkayae & Cirpa alkayae Vörös 25 \\
\hline andrasi & Spinostylosphaera andrasi Ozsvárt, Moix \& Kozur 15 \\
\hline angulocostatum & Procerithium? (Cosmocerithium?) angulocostatum Szabó 23 \\
\hline applanata & Globigerina applanata Hantken 27 \\
\hline aspinosa & Tamonella aspinosa Ozsvárt, Dumitrica \& Moix 17 \\
\hline atavus & Hystrix vinogradovi atavus Jánossy 46 \\
\hline barnabasi & Apringia barnabasi Vörös 25 \\
\hline & Capnuchosphaera barnabasi Kozur, Moix \& Ozsvárt 10 \\
\hline bersekensis & Phyllocrinus bersekensis Konieczyński, Pisera \& Főzy 27 \\
\hline bertalani & Callichirus bertalani Hyžný \& Müller 34 \\
\hline bifida & Sphenope bifida Vörös 26 \\
\hline bitnerae & Argyrotheca bitnerae Dulai in DulaI \& STACHACZ 34 \\
\hline borbalae & Capnuchosphaera borbalae Kozur, Moix \& Ozsvárt 10 \\
\hline bragini & Capnuchosphaera bragini Kozur, Moix \& Ozsvárt 10 \\
\hline & Tekinium bragini Ozsvárt, Dumitrica \& Hungerbühler in OzsvárT et al. 17 \\
\hline brevimanus & Callianassa brevimanus Beurlen 30 \\
\hline brev & $\begin{array}{l}\text { Caponabolella brevispinosa Ozsvárt, Dumitrica \& Hungerbühler in OzsvárT et } \\
\text { al. } 12\end{array}$ \\
\hline budaii & Stoppaniceras budaii Vörös 21 \\
\hline burrii & Hindeosphaera burrii Ozsvárt, Moix \& Kozur 13 \\
\hline capellinii & Ancyloceras capellini Matheron 26 \\
\hline carterae & Capnuchosphaera ottomanensis carterae Kozur, Moix \& Ozsvárt 11 \\
\hline catharinae & Prionorhynchia? catharinae Vörös 26 \\
\hline ciliciensis & Capnuchosphaera ciliciensis Kozur, Moix \& Ozsvárt 10 \\
\hline compactus & Poulpus compactus Ozsvárt, Dumitrica \& Hungerbühler in OzsvárT et al. 14 \\
\hline ovis & $\begin{array}{l}\text { Circopoulpus cornubovis Ozsvárt, Dumitrica \& Hungerbühler in OzsváRT et } \\
\text { al. } 12\end{array}$ \\
\hline coronata & Hungariella coronata Szabó 18 \\
\hline craterifera & Callianassa craterifera Lörenthey in LöRENTHEY \& BEURLEN 30 \\
\hline curvata & Weverella longispinosa curvata Kozur, Moix \& Ozsvárt 17 \\
\hline curvispina & $\begin{array}{l}\text { Annulohaeckeliella curvispina Ozsvárt, Dumitrica \& Hungerbühler in OzsvárT } \\
\text { et al. } 10\end{array}$ \\
\hline cylindrica & Capnuchosphaera cylindrica cylindrica Kozur, Moix \& Ozsvárt 11 \\
\hline daghestanicus & Perisphinctes daghestanicus Papp 24 \\
\hline danii & Laevitomaria danii Szabó 22 \\
\hline dentatus & Theodoxus (Calvertia?) grateloupianus dentatus Kókay 34 \\
\hline djani & Hindeosphaera djani Ozsvárt, Moix \& Kozur 13 \\
\hline doreckae & Apsidocrinus doreckae Konieczyński, Pisera \& Főzy 26 \\
\hline dulaii & Circopoulpus dulaii Ozsvárt, Dumitrica \& Hungerbühler in Ozsvárt et al. 12 \\
\hline dumitricai & Pseudostylosphaera dumitricai Ozsvárt, Moix \& Kozur 14 \\
\hline dunaii & Virgatosimoceras dunaii Scherzinger, Fözy \& Parent 25 \\
\hline ehiki & Allocricetus ébiki Schaub 46 \\
\hline elegans & Poulpus elegans Ozsvárt, Dumitrica \& Hungerbühler in Ozsvárt et al. 14 \\
\hline elegantula & Trochus (Mesotrochus) triadicus elegantula Kutassy 18 \\
\hline epuliformis & Proconulus epuliformis Szabó 23 \\
\hline
\end{tabular}




\begin{tabular}{|c|c|}
\hline estherae & Paronarhynchia estherae Vörös 26 \\
\hline fedori & Bakonybatrachus fedori Szentesi \& Venczel 35 \\
\hline fitosi & Magyarosuchus fitosi Ősi, Young, Galácz \& Rabi 37 \\
\hline fodori & Pneumatoraptor fodori Ösi, Apesteguía \& Kowalewski 46 \\
\hline fusiformis & Paramonocapnuchosphaera fusiformis Ozsvárt, Dumitrica \& Moix 14 \\
\hline galaczi & Eucycloidea galaczi Szabó 22 \\
\hline lobosa & Globigerina globosa Hantken 27 \\
\hline $\begin{array}{l}\text { goestlingensis } \\
\text { gombaszogensis }\end{array}$ & $\begin{array}{l}\text { Capnuchosphaera goestlingensis Kozur, Mostler \& Ozsvárt in Kozur et al. } 11 \\
\text { Ursus etruscus gombaszögensis Kretzoi } 47\end{array}$ \\
\hline racilispinosa & $\begin{array}{l}\text { Capnuchosphaera gracilispinosa gracilispinosa Kozur, Moix \& Ozsvárt } 11 \\
\text { Weverella gracilispinosa Kozur, Moix \& Ozsvárt } 17\end{array}$ \\
\hline randincisivus & Mastodon (Bunolophodon) grandincisivum Schlesinger 47 \\
\hline antkeni & Magellania hantkeni Meznerics 28 \\
\hline & Spinocapnuchosphaera hantkeni Kozur, Moix \& Ozsvárt 15 \\
\hline arzhauseri & Lautoconus harzhauseri Kovács in VICIÁN et al. 33 \\
\hline astata & Anoptychia hastata Szabó 22 \\
\hline hermanni & Stoppaniceras hermanni Vörös 21 \\
\hline hexaspinosa & Goricanella hexaspinosa Kozur, Mostler \& Ozsvárt in KozUR et al. 12 \\
\hline idasensis & Valvata hidasensis Kókay 34 \\
\hline hirmetzli & $\begin{array}{l}\text { Leptoconus hirmetzli Kovács \& Vicián } 33 \\
\text { Siratus hirmetzli Kovács } 33\end{array}$ \\
\hline hugluensis & Spinocapnuchosphaera tekini hugluensis Kozur, Moix \& Ozsvárt 15 \\
\hline ungarica & Cypraeorbis hungarica Schilder 29 \\
\hline hungaricus & $\begin{array}{l}\text { Absurdaster hungaricus Kroh, Lukeneder \& Gallemí } 27 \\
\text { Homarus hungaricus Tshudy, Hyžný, Dulai \& Jagt } 31\end{array}$ \\
\hline inaequispinosum & Tubospongopallium inaequispinosum Kozur, Moix \& Ozsvárt 17 \\
\hline inexpectatus & Pannoniasaurus inexpectatus Makádi, Caldwell \& Ösi 45 \\
\hline infrequens & Pelsochamops infrequens Makádi 46 \\
\hline ohansenae & Terebratulina johansenae Dulai 28 \\
\hline katalinae & Pugilina katalinae Kovács \& Vicián 29 \\
\hline kondai & Trochopsidea kondai Szabó 24 \\
\hline kozmai & Ajkaceratops kozmai Ösi, Butler \& Weishampel 36 \\
\hline kozuri & Poulpus kozuri Ozsvárt, Dumitrica \& Hungerbühler in Ozsvárt et al. 14 \\
\hline krocki & Merica krocki Kovács \& Vicián 29 \\
\hline liechtensteini & Stephanoceras liechtensteinii Papp 25 \\
\hline loczyi & Perisphinctes lóczyi Papp 24 \\
\hline lokutica & Rhapidothyris lokutica Vörös 26 \\
\hline longispinosa & $\begin{array}{l}\text { Caponabolella longispinosa Ozsvárt, Dumitrica \& Hungerbühler in Ozsvárt et } \\
\text { al. } 12\end{array}$ \\
\hline & Weverella longispinosa longispinosa Kozur, Moix \& Ozsvárt 17 \\
\hline longis & Minicrampus longispinosus Ozsvárt, Dumitrica \& Moix 13 \\
\hline longospinosus & $\begin{array}{l}\text { Silicarmiger longospinosus Ozsvárt, Dumitrica \& Hungerbühler in OzsváRT et } \\
\text { al. } 14\end{array}$ \\
\hline mandokii & Bathrotomaria mandokii Szabó 22 \\
\hline manyense & Cardium manyense Kókay 32 \\
\hline marginospinosa & Spinocapnuchosphaera tekini marginospinosa Kozur, Moix \& Ozsvárt 15 \\
\hline masseti & Spinostylosphaera masseti Ozsvárt, Dumitrica \& Moix 16 \\
\hline mersinensis & Capnuchosphaera mersinensis Kozur, Moix \& Ozsvárt 11 \\
\hline
\end{tabular}




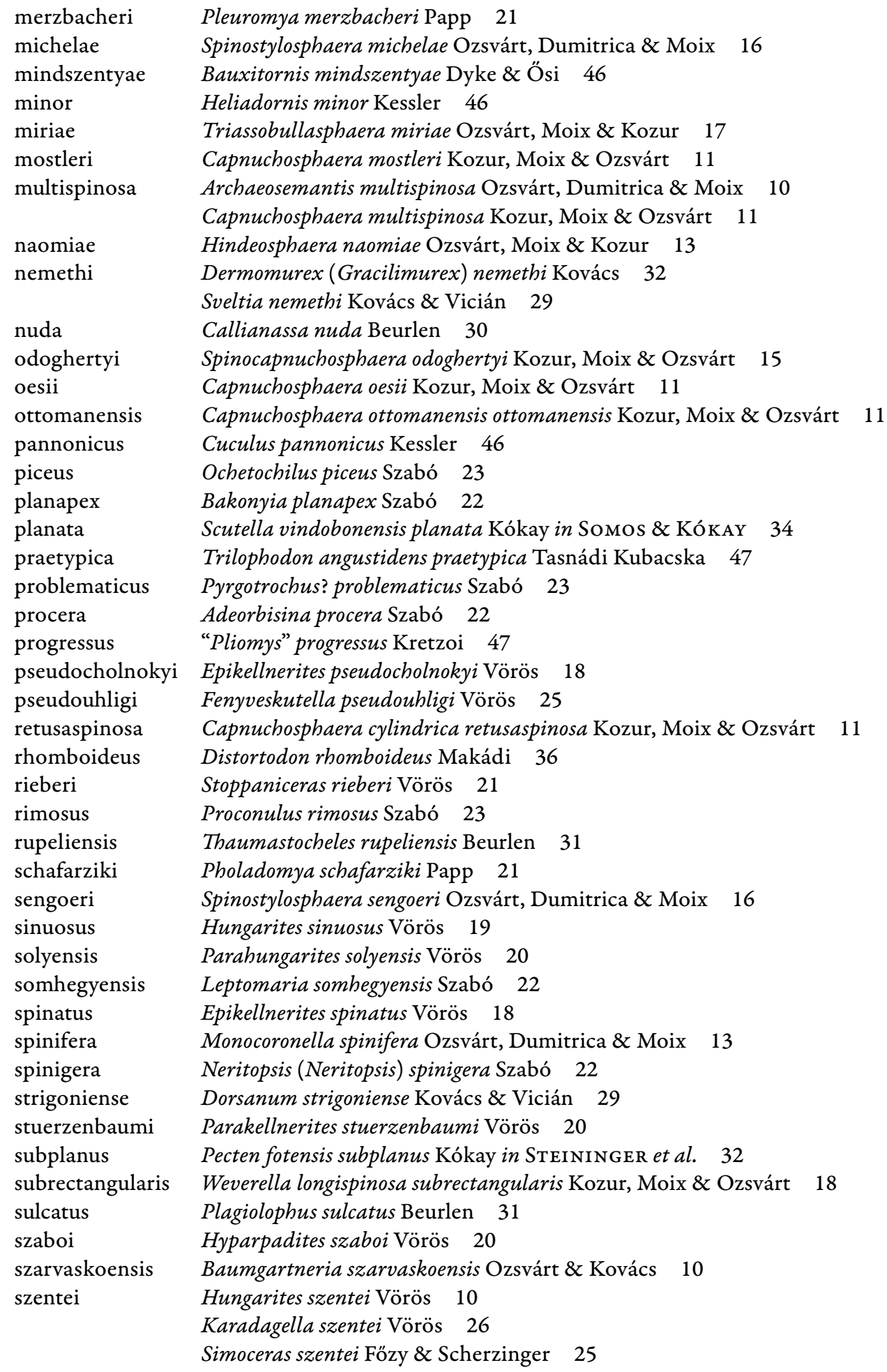




\begin{tabular}{|c|c|}
\hline szentei & Spinocapnuchosphaera szentei Kozur, Moix \& Ozsvárt 15 \\
\hline szilviae & Semicassis szilviae Kovács \& Vicián 33 \\
\hline szivesae & Spinocapnuchosphaera szivesae Kozur, Moix \& Ozsvárt 15 \\
\hline szobensis & Taurasia szobensis Csepreghy-Meznerics 33 \\
\hline szomodense & ?Chigaroceras szomodi Szives \& Főzy 26 \\
\hline szukacsi & Hungarobatrachus szukacsi Szentesi \& Venczel 35 \\
\hline tamasi & Epikellnerites tamasi Vörös 18 \\
\hline tekini & Spinocapnuchosphaera tekini tekini Kozur, Moix \& Ozsvárt 15 \\
\hline tenuispinosa & Paraweverella tenuispinosa Kozur, Moix \& Ozsvárt 14 \\
\hline theresiae & Fenyveskutella theresiae Vörös 25 \\
\hline tiliquoides & Chromatogenys tiliquoides Makádi \& Nydam 36 \\
\hline tortuospinosa & Capnuchosphaera tortuospinosa Kozur, Moix \& Ozsvárt 11 \\
\hline trabanti & Foxemys trabanti Rabi, Tong \& Botfalvai 36 \\
\hline triadicus & Trochus (Mesotrochus) triadicus Kutassy 18 \\
\hline tricuspidata & Spinocapnuchosphaera tricuspidata Kozur, Moix \& Ozsvárt 15 \\
\hline tridentata & Calappa tridentata Beurlen 29 \\
\hline trispinigera & Pietteia trispinigera Szabó 23 \\
\hline tumorspinosa & Capnuchosphaera tumorspinosa Kozur, Moix \& Ozsvárt 11 \\
\hline turcus & ?Pagurus turcus Müller 34 \\
\hline turkensis & Capnuchosphaera gracilispinosa turkensis Kozur, Moix \& Ozsvárt 11 \\
\hline tuvalica & Capnuchosphaera tuvalica Kozur, Moix \& Ozsvárt 12 \\
\hline unicarinatus & Dimorphotectus unicarinatus Szabó 22 \\
\hline uniseriatum & $\begin{array}{l}\text { Dorsanum (Dorsanum) nodosocostatum uniseriatum Kókay in STEININGER et } \\
\text { al. } 32\end{array}$ \\
\hline uromensis & Archidiskodon meridionalis ürömensis Vörös 46 \\
\hline vachardi & Spinostylosphaera vachardi Ozsvárt, Moix \& Kozur 16 \\
\hline vaszolyensis & Epikellnerites vaszolyensis Vörös 18 \\
\hline venusta & Spinocapnuchosphaera venusta Kozur, Moix \& Ozsvárt 15 \\
\hline vesicula & Ventricaria? vesicula Szabó 24 \\
\hline viciani & Euthria viciani Kovács 32 \\
\hline & Lepidochitona viciani Dell'Angelo, Sosso, Kroh \& Dulai 28 \\
\hline & Menathais viciani Kovács 33 \\
\hline vighi & Fenyveskutella vighi Vörös 25 \\
\hline vinczei & Nodihungarites vinczei Vörös 20 \\
\hline voeroesi & Tetracapnuchosphaera? voeroesi Kozur, Moix \& Ozsvárt 17 \\
\hline vorosi & Mochlodon vorosi Ösi, Prondvai, Butler \& Weishampel 42 \\
\hline yforma & Capnuchosphaera crassa yforma Kozur, Moix \& Ozsvárt 11 \\
\hline zagorseki & Rugia zagorseki Dulai 28 \\
\hline zircensis & Zircia zircensis Szabó 24 \\
\hline
\end{tabular}

\section{REFERENCES}

BÁLDi T. 1973: Mollusc fauna of the Hungarian Upper Oligocene (Egerian). Studies in stratigraphy, palaeoecology, palaeogeography and systematics. - Akadémiai Kiadó, Budapest, 511 pp. [Bál73]

Beurlen K. 1939: Neue Decapoden-Krebse aus dem ungarischen Tertiär. - Paläontologische Zeitschrift 21 (2): 135-160. https://doi.org/10.1007/bf03043252 [Beu39] 
Bitner M. A., Dulai A. \& Galácz A. 2011: Middle Eocene brachiopods from the Szőc Limestone Formation (Bakony Mountains, Hungary), with a description of a new genus. - Neues Jabrbuch für Geologie und Paläontologie, Abhandlungen 259(1): 113-128. https://doi.org/10.1127/0077-7749/2010/0113 [Bit11]

Busulini A., Beschin C. \& Tessier G. 2014: A re-evaluation of extinct European crabs referred to the genus Calappilia A. Milne Edwards in Bouillé, 1873 (Brachyura, Calappidae). - In: FraAije R. H. B., HyžnÝ M., Jagt J. W. M., Krobicki M. \& Van Bakel B. W. M. (eds): Proceedings of the 5th Symposium on Mesozoic and Cenozoic Decapod Crustaceans, Krakow, Poland, 2013: A tribute to Pál Mihály Müller. Scripta Geologica 147: 193-219. [Bus14]

Conti M. A. \& Szabó J. 1987: Comparison of Bajocian gastropod faunas from the Bakony Mts. (Hungary) and Umbria (Italy). - Annales historico-naturales Musei nationalis hungarici 79: 43-59. [Con87]

CsÁszÁR G. 1997: Basic litostratigraphic units of Hungary. Charts and short descriptions. - Geological Institute of Hungary, Budapest, $114 \mathrm{pp}$.

Csepreghy-Meznerics I. 1956: A szobi és letkési puhatestű fauna. (Die Molluskenfauna von Szob und Letkés.) - Magyar Állami Földtani Intézet Évkönyve (Annals of the Hungarian Geological Institute) 45(2): 363-477. [C-M56]

Dell'Angelo B., Sosso M., Kroh A. \& Dulai A. 2015: Polyplacophora from the Eocene of Gánt, Hungary. - Bulletin of Geosciences 90(2): 359-370. https://doi.org/10.3140/bull.geosci.1517 [Del15]

Dulai A. 2011: Late Eocene (Priabonian) micromorphic brachiopods from the Upper Austrian Molasse Zone. - Memoirs of the Association of Australasian Palaeontologists 41: 295-313. [Dul11a]

Dulai A. \& STACHaCz M.2011: New Middle Miocene Argyrotheca (Brachiopoda; Megathyrididae) species from the Central Paratethys. - Földtani Közlöny (Bulletin of the Hungarian Geological Society) 141(3): 283-291. [Dul11b]

Dyke G. J. \& Ösı A. 2010: A review of Late Cretaceous fossil birds from Hungary. - Geological Journal 45(4): 434-444. https://doi.org/10.1002/gj.1209 [Dyk10]

FöZY I. \& SCHERZINGER A. 2011: Simoceras szentei n. sp., a new ammonite species from the lowermost Tithonian of the Gerecse Mountains (Hungary) - the earliest record of the genus. Neues Jahrbuch für Geologie und Paläontologie, Abhandlungen 262(1): 117-127. https://doi.org/10.1127/0077-7749/2011/0195 [Föz11]

FöZY I. \& SCHERZINGER A. 2013: Systematic descriptions of Tithonian ammonites of the Gerecse Mountains. - In: Fözy I. (ed.): Late Jurassic - Early Cretaceous fauna, biostratigraphy, facies and deformation history of the carbonate formations in the Gerecse and Pilis Mountains (Transdanubian Range, Hungary). Institute of Geosciences, University of Szeged, GeoLitera Publishing House, pp. 207-292. [Főz13]

Galácz A. \& Szente I. 2008: Middle Jurassic fossils from Daghestan. A revision of ammonites and bivalves collected by the Déchy Caucasus expeditions (1884-1902). - Hantkeniana 6: 109-125. [Gal08]

GASPARIK M. 2007: "Elephants" in the cellar. A revision of the Neogene proboscidean remains, damaged in the fire of the Hungarian Natural History Museum in 1956. - Fragmenta Palaeontologica Hungarica 24-25: 83-91. [Gas07]

GASPARIK M. \& MAR KOv G. N. 2009: Gomphotherium 'annectens group' (Proboscidea) in Hungary. - Fragmenta Palaeontologica Hungarica 27: 73-79. [Gas09]

Gatto R., Monari S., Szabó J. \& Conti M. A. 2015: The Jurassic pleurotomarioidean gastropod Laevitomaria and its palaeobiogeographical history. - Acta Palaeontologica Polonica 60(1): 217-233. https://doi.org/10.4202/app.2013.0012 [Gat15] 
Gradstein F. M., Ogg J. G., Schmitz M. D. \& Ogg, G. M. (eds) 2012: The geologic time scale 2012. - Elsevier, Amsterdam, 1144 pp. https://doi.org/10.1016/c2011-1-08249-8

HABly L., ERdei B. \& KVAČEK Z. 2001: 19th century's palaeobotanical types and originals of the Hungarian Natural History Museum. - Studia Naturalia 13: 1-235.

HABly L. \& SZAKÁly M. 1989: The catalogue of leaf-fossil types preserved in Hungary. - Akadémiai Kiadó, Budapest, 253 pp.

Hant Ken M. 1883a: Die Clavulina Szabói-Schichten im Gebiete der Euganeen und der Meeralpen und die cretacische Scaglia in den Euganeen. - Mathematische und Naturwissenschaftliche Berichte aus Ungarn 2: 121-169. [Han83a]

HantKen M. 1883b: A Clavulina Szabói-rétegek, az Euganeák és a tengeri Alpok területén és a krétakoru „Scaglia” az Euganeákban. - Értekezések a Természettudományok Köréből 13: 1-47. [Han83b]

Harzhauser M. \& Kowalke T. 2004: Survey of the nassariid gastropods in the Neogene Paratethys (Mollusca: Caenogastropoda: Buccinoidea). - Archiv für Molluskenkunde 133(1-2): 1-61. https://doi.org/10.1127/arch.moll/133/2004/1 [Har04]

Harzhauser M. \& Landau B. 2016: A revision of the Neogene Conidae and Conorbidae (Gastropoda) of the Paratethys Sea. - Zootaxa 4210(1): 1-178. https://doi.org/10.11646/zootaxa.4210.1.1 [Har16]

HYŽNÝ M. 2016: Diversity and distribution patterns of the Oligocene and Miocene decapod crustaceans (Crustacea: Malacostraca) of the Western and Central Paratethys. - Geologica Carpathica 67(5): 471-494. https://doi.org/10.1515/geoca-2016-0030 [Hyž16]

HyžNÝ M. \& Dulai A. 2014: Deep-water fossorial shrimps from the Oligocene Kiscell Clay of Hungary: Taxonomy and palaeoecology. - Acta Palaeontologica Polonica 59(4): 947-965. https://doi.org/10.4202/app.2012.0078 [Hyž14]

HYŽNÝ M. \& KLOMPMAKER A. A. 2015: Systematics, phylogeny, and taphonomy of ghost shrimps (Decapoda): a perspective from the fossil record. - Arthropod Systematics \& Phylogeny 73(3): 401-437. [Hyž15]

HyžNÝ M. \& Müller P. M. 2010: The first fossil record of the genus Callichirus (Decapoda, Axiidea, Callianassidae) from the middle Miocene of Hungary, with description of a new species. - Bulletin of the Mizunami Fossil Museum 36: 37-43. [Hyž10]

International Commission on Zoological Nomenclature 1999: International code of zoological nomenclature. - The International Trust for Zoological Nomenclature, London, 306 pp. https://doi.org/10.5962/bhl.title.50608

JÁnossy D. 1972: Ein kleiner Hystrix aus Altpleistozän der Fundstelle Osztramos 8. (Nordungarn). - Vertebrata Hungarica 13: 163-182. [Ján72]

Jánossy D. 1986: Pleistocene Vertebrate Faunas of Hungary. - Akadémiai Kiadó, Budapest, 208 pp. https://doi.org/10.1016/s0920-5446(08)x7001-3

KESSLER J. 2009: Új eredmények a Kárpát-medence neogén és negyedidőszaki madárvilágához, I. (New results with regard to the Neogene and Quaternary Avifauna of the Carpathian Basin, Part I.) - Földtani Közlöny (Bulletin of the Hungarian Geological Society) 139(1): 67-81. [Kes09]

KESSLER J. 2010: Új eredmények a Kárpát-medence neogén és negyedidőszaki madárvilágához III. (New results with regard to the Neogene and Quaternary Avifauna of the Carpathian Basin, Part III.) - Földtani Közlöny (Bulletin of the Hungarian Geological Society) 140(1): 53-71. [Kes10]

Klein J., Busnardo R., Company M., Delanoy G., Kakabadze M., Reboulet S., Ropolo P., VAŠíčeK Z. \& Vermeulen J. 2007: Lower Cretaceous Ammonites III. Bochianitoidea, Pro- 
tancyloceratoidea, Ancyloceratoidea, Ptychoceratoidea. - In: Riggraf W. (ed.): Fossilium Catalogus I: Animalia 144: 1-381. Backhuys Publishers, Leiden. [Kle07]

KóKAY J. 1967: A Bakony-hegység felsőtortonai képződményei. (Obertortonische Ablagerungen des Bakonygebirges.) - Földtani Közlöny (Bulletin of the Hungarian Geological Society) 97(1): 74-90. [Kók67]

KóKAY J. 2006: Nonmarine mollusc fauna from the Lower and Middle Miocene, Bakony Mts, W Hungary. - Geologica Hungarica, Series Palaeontologica 56: 1-196. [Kók06]

Konidaris G. E., Roussiakis S. J., Theodorou G. E. \& Koufos G. D. 2014: The Eurasian occurrence of the shovel-tusker Konobelodon (Mammalia, Proboscidea) as illuminated by its presence in the late Miocene of Pikermi (Greece). - Journal of Vertebrate Paleontology 34(6): 1437-1453. https://doi.org/10.1080/02724634.2014.873622 [KRT14]

Konieczyński K., Pisera A. \& Fözy I. 2016: Early Cretaceous cyrtocrinids (Crinoidea) from the Gerecse Mountains, northern Hungary. - Neues Jabrbuch für Geologie und Paläontologie, Abhandlungen 279(2): 155-166. https://doi.org/10.1127/njgpa/2016/0547 [KPF16]

Kovács Z. 2018a: Description of three new species of Muricidae (Neogastropoda) from the Miocene Paratethys. - Novapex 19(1): 29-35. [Kov18a]

Kovács Z. 2018b: New records of the genus Euthria (Mollusca, Buccinidae) in the Miocene Paratethys. - Földtani Közlöny (Bulletin of the Hungarian Geological Society) 148(2): 179-182. https://doi.org/10.23928/foldt.kozl.2018.148.2.179 [Kov18b]

Kovács Z. \& Vicián Z. 2014: Badenian (Middle Miocene) Conoidean (Neogastropoda) fauna from Letkés (N Hungary). - Fragmenta Palaeontologica Hungarica 30: 53-100. [Kov14]

Kovács Z. \& ViciÁn Z. 2016: A new Egerian (Upper Oligocene - Lower Miocene) gastropod fauna from the Esztergom Basin (NE Transdanubia, Hungary). - Földtani Közlöny (Bulletin of the Hungarian Geological Society) 146(3): 233-255. [Kov16]

Kovács Z. \& VicıÁn Z. 2017: Middle Miocene Tonnoidea and Ficoidea (Caenogastropoda) assemblages from Letkés (Hungary). - Fragmenta Palaeontologica Hungarica 34: 75-104. https://doi.org/10.17111/fragmpalhung.2017.34.75 [Kov17]

Kozur H. W., Moix P. \& Ozsvárt P. 2009: New Spumellaria (Radiolaria) from the early Tuvalian Spongotortilispinus moixi Zone of Southeastern Turkey, with some remarks on the age of this fauna. - Jahrbuch der Geologischen Bundesanstalt 149(1): 25-59. [Koz09]

Kretzor M. 1938: Die Raubtiere von Gombaszög nebst einer Übersicht der Gesamtfauna. (Ein Beitrag zur Stratigraphie des Altquartaers.) - Annales historico-naturales Musei nationalis bungarici 31: 88-157. [Kre38]

KRETzor M. 1969: A magyarországi quarter és pliocén szárazföldi biosztratigráfiájának vázlata. (Sketch of the Late Cenozoic (Pliocene and Quaternary) terrestrial stratigraphy of Hungary.) - Földrajzi Közlemények (Geographical Review) 17(3): 179-204.

Kroh A., Lukeneder A. \& Gallemí J. 2014: Absurdaster, a new genus of basal atelostomate from the Early Cretaceous of Europe and its phylogenetic position. - Cretaceous Research 48: 235-249. https://doi.org/10.1016/j.cretres.2013.11.013 [Kro14]

Kutassy A. 1927: Beiträge zur Stratigraphie und Paläontologie der alpinen Triasschichten in der Umgebung von Budapest. - Magyar Királyi Földtani Intézet Évkönyve (Mitteilungen aus dem Jahrbuche der Königlichen Ungarischen Geologischen Anstalt) 27(2): 107-175. [Kut27]

KuTAssy E. 1933: Újabb adatok a Budapest-környéki dachsteini mészkő faunájának ismeretéhez. (Weitere Beiträge zur Kenntnis der Fauna des Dachsteinkalkes in der Umgebung von Budapest.) - Matematikai és Természettudományi Értesitö (Mathematischer und Naturwissenschaftlicher Anzeiger der Ungarischen Akademie der Wissenschaften) 49: 222-250. [Kut33]

LöRENTHEY E. \& BEURLEN K. 1929: Die fossilen Dekapoden der Länder der Ungarischen Krone. - Geologica Hungarica, Series Palaeontologica 3: 1-420. [Lőr29] 
MAKÁDi L. 2013a: A new polyglyphanodontine lizard (Squamata: Borioteiioidea) from the Late Cretaceous Iharkút locality (Santonian, Hungary). - Cretaceous Research 46: 166-176. https://doi.org/10.1016/j.cretres.2013.08.001 [Mak13a]

MAKÁDi L. 2013b: The first known chamopsiid lizard (Squamata) from the Upper Cretaceous of Europe (Csehbánya Formation; Hungary, Bakony Mts). - Annales de Paléontologie 99(3): 261-274. https://doi.org/10.1016/j.annpal.2013.07.002 [Mak13b]

MaKÁdi L., Caldwell M. W. \& Ösi A. 2012: The first freshwater mosasauroid (Upper Cretaceous, Hungary) and a new clade of basal mosasauroids. - PLoS ONE 7(12): e51781. https://doi.org/10.1371/journal.pone.0051781 [Mak12]

MAKÁDi L. \& NydAM R. L. 2015: A new durophagous scincomorphan lizard genus from the Late Cretaceous Iharkút locality (Hungary, Bakony Mts). - Paläontologische Zeitschrift 89(4): 925-941. https://doi.org/10.1007/s12542-014-0253-1 [Mak15]

Matheron P. 1878-1880: Recherches paléontologiques dans le Midi de la France. - Marseille. [Mat80]

MeZnerics I. 1944: Die Brachiopoden des ungarischen Tertiärs. - Annales historico-naturales Musei nationalis hungarici 36: 10-60. [Mez44]

MÜLLER P. 1984: A bádeni emelet tízlábú rákjai. (Decapod Crustacea of the Badenian.) - Geologica Hungarica, Series Palaeontologica 42: 1-317. [Mül84]

Ozsvárt P., Dumitrica P., HungerbüHler A. \& Moix P. 2017a: Mono- and dicyrtid Nassellaria (Radiolaria) from the Upper Carnian of the Sorgun Ophiolitic Mélange, Southern Turkey and Kopría Mélange, Rhodes, Greece. - Revue de Micropaléontologie 60(1): 137-160. https://doi.org/10.1016/j.revmic.2016.11.004 [Ozs17a]

Ozsvá RT P., Dumitrica P. \& Moix P. 2017b: New early Tuvalian (Carnian, Triassic) radiolarians from the Huğlu-Pindos succession in the Sorgun Ophiolitic Mélange, Southern Turkey. Ofioliti 42(1): 55-67. https://doi.org/10.4454/ofioliti.v42i2.448 [Ozs17b]

OzsváRT P. \& Kovács S. 2012: Revised Middle and Late Triassic radiolarian ages for ophiolite mélanges: implications for the geodynamic evolution of the northern part of the early Mesozoic Neotethyan subbasins. - Bulletin de la Société géologique de France 183(4): 273-286. https://doi.org/10.2113/gssgfbull.183.4.273 [Ozs12]

Ozsvárt P., Moix P. \& Kozur H. W. 2015: New Carnian (Upper Triassic) radiolarians from the Sorgun Ophiolitic Mélange, southern Turkey. - Neues Jahrbuch für Geologie und Paläontologie, Abhandlungen 277(3): 337-352. https://doi.org/10.1127/njgpa/2015/0509 [Ozs15]

Ősi A., Apesteguía S. \& Kowalewski M. 2010a: Non-avian theropod dinosaurs from the early Late Cretaceous of central Europe. - Cretaceous Research 31(3): 304-320. https://doi.org/10.1016/j.cretres.2010.01.001 [Ösi10a]

Ösi A., Butler R. J. \& Weishampel D. B. 2010b: A Late Cretaceous ceratopsian dinosaur from Europe with Asian affinities. - Nature 465: 466-468. https://doi.org/10.1038/nature09019 [Ösi10b]

Ösi A., Prondvai E., Butler R. \& Weishampel D. B. 2012: Phylogeny, histology and inferred body size evolution in a new rhabdodontid dinosaur from the Late Cretaceous of Hungary. PLOS ONE 7(9): e44318. https://doi.org/10.1371/journal.pone.0044318 [Ösi12]

Ösi A., Young M. T., GALÁcz A. \& RABi M.2018: A new large-bodied thalattosuchian crocodyliform from the Lower Jurassic (Toarcian) of Hungary, with further evidence of the mosaic acquisition of marine adaptations in Metriorhynchoidea. - PeerJ 6(1): e4668. https://doi.org/10.7717/peerj.4668 [Ösi18]

PÁLFY J. 2009: Review of invertebrate and vertebrate paleontological types in the collection of the Hungarian Natural History Museum. - Annales historico-naturales Musei nationalis hungarici 101: 5-22. 
Pálfy J., Dulai A., Gasparik M., Ozsvárt P., Pazonyi P. \& Szives O. 2008: Catalogue ofinvertebrate and vertebrate paleontological type specimens of the Hungarian Natural History Museum. - Hungarian Natural History Museum, Budapest, 209 pp.

PAPP K. 1907: Beschreibung der während der Forschungsreisen M. v. Déchys im Kaukasus gesammelten Versteinerungen. - In: DÉcHy M. (ed.): Kaukasus. Reisen und Forschungen im kaukasischen Hochgebirge. Band III. Dietrich Reimer Verlag, Berlin, pp. 141-173. [Pap07]

Piller W. E., Harzhauser M. \& Mandic O. 2007: Miocene Central Paratethys stratigraphy current status and future directions. - Stratigraphy 4(2-3): 151-168.

Rabi M., Tong H. \& Botfalvai G. 2012: A new species of the side-necked turtle Foxemys (Pelomedusoides: Bothremydidae) from the Late Cretaceous of Hungary and the historical biogeography of the Bothremydini. - Geological Magazine 149(4): 662-674. https://doi.org/10.1017/s0016756811000756 [Rab12]

Raven J. G. M. 2016: Notes on molluscs from NW Borneo. 3. A revision of Taurasia (Gastropoda, Muricidae) and Preangeria (Gastropoda, Buccinidae) with comments on Semiricinula from NW Borneo. - Vita Malacologica 15: 77-104. [Rav16]

Schau B S. 1930: Quartäre und jungtertiäre Hamster. - Abhandlungen der Schweizerischen Palaeontologischen Gesellschaft 49: 1-49. [ScS30]

Scherzinger A., Fözy I. \& Parent H. 2010: The Early Tithonian (Late Jurassic) ammonite genus Virgatosimoceras Spath (Ammonoidea: Simoceratidae) - revision and value for correlation. - Neues Jahrbuch für Geologie und Paläontologie, Abhandlungen 256(2): 195-212. https://doi.org/10.1127/0077-7749/2010/0047 [ScA10]

Schilder F. A. 1932: Neue fossile Cypraeacea (Moll. Gastr.). - Sitzungsberichte der Gesellschaft Naturforschender Freunde zu Berlin 1932: 254-269. [ScF32]

SCHLesinger G. 1917: Die Mastodonten des K. K. Naturhistorischen Hofmuseums. Morphologisch-phylogenetische Untersuchungen. - Denkschriften des K. K. Naturhistorischen Hofmuseums 1: 1-230. [ScG17]

SCHLESINGer G. 1922: Die Mastodonten der Budapester Sammlungen. (Untersuchungen über Morphologie, Phylogenie, Ethologie und Stratigraphie europäischer Mastodonten.) - Geologica Hungarica 2(1): 1-284. [ScG22]

Somos L. \& KóKAY J. 1960: Földtani megfigyelések a Mecsekhegységi liászban és miocénben. (Geologische Beobachtungen im Lias und Miozän des Mecsekgebirges.) - Földtani Közlöny (Bulletin of the Hungarian Geological Society) 90(3): 331-347. [Som60]

Steininger F., Čtrroký P., Hölzl O., Kókay J., Schlickum W. R., Schultz O. \& Strauch F. 1973: Die Molluskenfaunen des Ottnangien. - In: SENEš J. (ed.): Chronostratigraphie und Neostratotypen. Miozän der zentralen Paratethys. Bd. III. M2 Ottnangien. Die Innviertler, Salgótarjáner, Bántapusztaer Schichtengruppe und die Rzehakia Formation. Verlag der Slowakischen Akademie der Wissenschaften, Bratislava, pp. 380-615. [Ste73]

SzABÓ J. 1980: Lower and Middle Jurassic gastropods from the Bakony Mountains (Hungary). Part II. Pleurotomariacea and Fissurellacea (Archaeogastropoda). - Annales historico-naturales Musei nationalis hungarici 72: 49-71. [Sza80]

SzABó J. 1981: Lower and Middle Jurassic gastropods from the Bakony Mountains (Hungary). Part III. Patellacea and Trochacea (Archaeogastropoda). - Annales historico-naturales Musei nationalis hungarici 73: 55-67. [Sza81]

SzABó J. 1982: Lower and Middle Jurassic gastropods from the Bakony Mountains (Hungary). Part IV: Neritacea, Craspedostomatacea, Amberleyacea (Archaeogastropoda). - Annales historico-naturales Musei nationalis hungarici 74: 17-33. [Sza82] 
Szabó J. 1983: Lower and Middle Jurassic gastropods from the Bakony Mountains (Hungary). Part V. Supplement to Archaeogastropoda; Caenogastropoda. - Annales historico-naturales Musei nationalis hungarici 75: 27-46. [Sza83]

SzABó J. 2009: Gastropods of the Early Jurassic Hierlatz Limestone Formation; part 1: a revision of type collections from Austrian and Hungarian localities. - Fragmenta Palaeontologica Hungarica 26: 1-108. [Sza09]

SzABó J. 2011: Corrections to three gastropod genera, established by Kutassy on Late Triassic type species from Dachstein Limestone localities of Budapest (Hungary). - Neues Jabrbuch für Geologie und Paläontologie, Abhandlungen 261(1): 37-47. https://doi.org/10.1127/0077-7749/2011/0145 [Sza11]

Szentesi Z. \& Venczel M. 2010: An advanced anuran from the Late Cretaceous (Santonian) of Hungary. - Neues Jahrbuch für Geologie und Paläontologie, Abhandlungen 256(3): 291-302. https://doi.org/10.1127/0077-7749/2010/0054 [Sze10]

Szentesi Z. \& VenCzel M. 2012: A new discoglossid frog from the Upper Cretaceous (Santonian) of Hungary. - Cretaceous Research 34: 327-333. https://doi.org/10.1016/j.cretres.2011.11.012 [Sze12]

SzIves O. \& FöZy I. 2013: Systematic descriptions of Early Cretaceous ammonites of the carbonate formations of the Gerecse Mountains, Hungary. - In: Fözy I. (ed.): Late Jurassic - Early Cretaceous fauna, biostratigraphy, facies and deformation history of the carbonate formations in the Gerecse and Pilis Mountains (Transdanubian Range, Hungary). Institute of Geosciences, University of Szeged, GeoLitera Publishing House, pp. 293-342. [Szi13]

Sztrákos K. 1973: Révision des espèces “Globigerina” applanata et "Globigerina” globosa décrites par M. Hantken d'Euganea (Italie). - Revue de Micropaléontologie 16(4): 224-228. [Szt73]

TASNÁDI KuBACSKA A. 1939: Trilophodon angustidens Cuv. forma praetypica koponyamaradványa Zagyvapálfalváról. (Ein primitiver Vertreter des Formenkreises Trilophodon angustidens Cuv. aus Ungarn.) - Annales historico-naturales Musei nationalis hungarici 32: 154-164. [Tas39]

Tshudy D., HyžnÝ M., Dulai A. \& JAGT J. W. M. 2018: Appraisal of the fossil record of Homarus (nephropid lobster), with description of a new species from the Upper Oligocene of Hungary and remarks on the status of Hoploparia. - Journal of Paleontology 92(2): 170-182. https://doi.org/10.1017/jpa.2017.65 [Tsh18]

Vicián Z., KRock H. \& KovÁcs Z. 2017: New gastropod records from the Cenozoic of Hungary. - Földtani Közlöny (Bulletin of the Hungarian Geological Society) 147(3): 265-281. https://doi.org/10.23928/foldt.kozl.2017.147.3.265 [Vic17]

VirÁg A. 2009: Revision of the "Archidiskodon meridionalis ürömensis" Vörös, 1979 in the context of the mammoth evolution in Eurasia. - Central European Geology 52(3-4): 405-419. https://doi.org/10.1556/ceugeol.52.2009.3-4.11 [Vir09]

VIRÁG A. \& Ösı A. 2017: Morphometry, microstructure, and wear pattern of neornithischian dinosaur teeth from the Upper Cretaceous Iharkút locality (Hungary). - The Anatomical Record 300(8): 1439-1463. https://doi.org/10.1002/ar.23592 [Vir17]

Vörös A. 2009: The Pliensbachian brachiopods of the Bakony Mountains (Hungary). - Geologica Hungarica, Series Palaeontologica 58: 1-300. [Vör09]

VöRös A. 2013: Latest Jurassic - earliest Cretaceous brachiopods from the Gerecse and Pilis Mountains (Hungary). - In: Fözy I. (ed.): Late Jurassic - Early Cretaceous fauna, biostratigraphy, facies and deformation history of the carbonate formations in the Gerecse and Pilis Mountains (Transdanubian Range, Hungary). Institute of Geosciences, University of Szeged, GeoLitera Publishing House, pp. 377-408. [VöA13] 
Vörös A. 2014a: Sphenope, a new genus of Pygopidae (Terebratulida, Brachiopoda) from the Mediterranean Late Jurassic and Early Cretaceous. - Fragmenta Palaeontologica Hungarica 30: 1-14. [VöA14a]

VöRös A. 2014b: Early Jurassic brachiopods from diverse localities of northern Anatolia (Turkey). - Fragmenta Palaeontologica Hungarica 31: 7-49. https://doi.org/10.17111/fragmpalhung.2014.31.7 [VöA14b]

Vörös A. 2018: The Upper Anisian ammonoids of the Balaton Highland (Middle Triassic, Hungary). - Geologica Hungarica, Series Palaeontologica 60: 1-241. [VöA18]

VöRös I. 1979: Archidiskodon meridionalis ürömensis n. ssp. from the Lower Pleistocene of the Carpathian Basin. - Fragmenta Mineralogica et Palaeontologica 9: 5-8. [VöI79]

WAgner J. \& GASPARIK M. 2014: Research history of Pleistocene faunas in Gombasek quarry (Slovakia), with comments to the type specimen and the type locality of Ursus deningeri gombaszogensis Kretzoi, 1938. - Fragmenta Palaeontologica Hungarica 31: 125-143.

https://doi.org/10.17111/fragmpalhung.2014.31.125 [Wag14] 